\title{
R-Loops between nascent pri-miRNAs and the encoding loci promote co- transcriptional processing of miRNAs in plants.
}

Lucia Gonzalo ${ }^{1,7}$, lleana Tossolini ${ }^{1,7}$, Tomasz Gulanicz ${ }^{2,3,7}$, Damian A. Cambiagno $^{1,6}$, Anna Kasprowicz-Maluski ${ }^{4}$, Jan Dariusz Smolinski ${ }^{3}$, Sebastian Marquardt $^{5}$, Zofia Szweykowska-Kulinska ${ }^{2}$, Artur Jarmolowski2 ${ }^{*}$, Pablo A. Manavella $^{1^{*}}$

${ }^{1}$ Instituto de Agrobiotecnología del Litoral (CONICET-UNL), Cátedra de Biología Celular y Molecular, Facultad de Bioquímica y Ciencias Biológicas, Universidad Nacional del Litoral, 3000 Santa Fe, Argentina.

${ }^{2}$ Department of Gene Expression, Institute of Molecular Biology and Biotechnology, Adam Mickiewicz University, Poznan 61-614, Poland.

${ }^{3}$ Centre For Modern Interdisciplinary Technologies, Nicolaus Copernicus University, Torun 87-100, Poland.

${ }^{4}$ Department of Molecular and Cellular Biology, Institute of Molecular Biology and Biotechnology, Adam Mickiewicz University, Poznan 61-614, Poland.

${ }^{5}$ Copenhagen Plant Science Centre, Department of Plant and Environmental Sciences, University of Copenhagen, 1870 Frederiksberg C, Denmark.

${ }^{6}$ Current address: Unidad de Estudios Agropecuarios (UDEA), INTA-CONICET, Córdoba, Argentina.

${ }^{7}$ These authors contributed equally

*To whom correspondence should be addressed. E-mail pablomanavella@ial.santafe-conicet.gov.ar; artjarmo@amu.edu.pl

\section{Summary}

In most organisms, the maturation of nascent RNAs is coupled to transcription, undergoing many processing steps co-transcriptionally. Unlike in animals, the RNA polymerase II (RNAPII) transcribes microRNAs (miRNAs) as long and structurally variable pri-miRNAs in plants. Current evidence suggests that the miRNA biogenesis complex assembly initiates early during the transcription of pri-miRNAs in plants. However, it is unknown whether miRNA processing occurs co-transcriptionally. Here, we show that plant miRNA biogenesis is coupled to transcription in a process that relies on the formation of DNA:RNA hybrids (R- 
loops) between the nascent transcript and the encoding loci. We used native elongating transcript sequencing data and imaging techniques to demonstrate that plant miRNA biogenesis occurs co-transcriptionally. We found that the entire biogenesis occurs coupled to transcription for pri-miRNAs processed from the loop but requires a second nucleoplasmic step for those processed from the base of the hairpin. Furthermore, we found that co- and post-transcriptional miRNA processing mechanisms co-exist for most miRNAs in a dynamic balance. Notably, we discovered that R-loops between the 5 '-end single-stranded arm of the pri-miRNAs and the encoding loci anchor the transcript, promoting cotranscriptional processing. Our data demonstrate the coupling of transcription and miRNA processing in plants and discovered an unexpected function for Rloops promoting RNA processing. Furthermore, our results suggest the neofunctionalization of co-transcriptionally processed miRNAs, boosting countless regulatory scenarios.

\section{Introduction}

The biogenesis of miRNAs in plants is a unique and evolutionary divergent pathway that differs from its counterpart in metazoans (Axtell et al., 2011; Zhang et al., 2018). In plants, for example, independent transcriptional units containing specific promoters, terminator and even introns encode most miRNAs (Cuperus et al., 2011; Szarzynska et al., 2009). The RNA polymerase II (RNAPII) transcribes plant MIRNA loci as capped and polyadenylated primary transcripts (pri-miRNAs). Unlike the animal pathway, where DROSHA and DICER concatenate to produce the mature miRNAs, a single processing complex containing DICER-Like 1 (DCL1) conducts the entire process inside the plant nucleus (Achkar et al., 2016; Bartel, 2018).

Opposite to pri-miRNAs in metazoans, which are homogeneous in size, plant's pri-miRNAs are highly variable in length and secondary structure ranging from hundreds to thousands of base pairs in polycistronic transcripts (Bologna et al., 2013; Moro et al., 2018; Singh et al., 2020). This particularity confronts DCL1 with a problem: recognizing the position of the active miRNA within such variable pri-miRNAs. Consequently, the miRNA-processing complex of plants relies on accessory proteins, such as HYPONASTIC LEAVES1 (HYL1) and SERRATE $(\mathrm{SE})$, and structural features in the pri-miRNAs to guide DCL1 to the precise 
slicing positions \{Dong, 2008 \#13;Re, 2019 \#14;Rojas, 2020 \#120;Yang, 2014 $\# 16\}$. As a result, alternative processing modes take place depending on the characteristics of each pri-miRNA (Moro et al., 2018; Zhu et al., 2013). In most cases, DCL1 produces a first cut near the base of the hairpin structure in a process known as Base-to-Loop processing (BTL) that resembles the processing from pri- to pre-miRNA by Drosha in animals (Moro et al., 2018). In other cases, the processing complex recognizes features in the terminal loop and initiates DCL1-mediated processing from the hairpin loop to the base (LTB) (Addo-Quaye et al., 2009; Bologna et al., 2009; Bologna et al., 2013; Moro et al., 2018; Zhu et al., 2013). Interestingly, sequential cuts of the pri-miRNA every $\sim 21$ nt by DCL1 releases the mature miRNA from long precursors (BTLs and LTBs) (Bologna et al., 2013; Moro et al., 2018).

Some components of the miRNA-biogenesis complex, such as DCL1 and HYL1, are located in nuclear speckles known as dicing bodies (D-Bodies) but alto associated with the MIRNA loci (Bhat et al., 2020; Cambiagno et al., 2021; Fang et al., 2015; Kim et al., 2011; Wang et al., 2013). The recruitment of DCL1 to the MIRNA loci relies on its association with the RNAPII-accessory complexes MEDIATOR and ELONGATOR. In the first case, HASTY (HST), the plant ortholog of human EXPORTIN 5, acts as a scaffold stabilizing the interaction of MED37-DCL1 that allows the recruitment of DCL1 to nascent pri-miRNAs (Cambiagno et al., 2021). Similarly, DCL1 recruitment to the MIRNA relies on the elongator complex (Fang et al., 2015). Such recruitment of the processing machinery to MIRNA loci may suggest that miRNA biogenesis takes place cotranscriptionally in plants, as described for animals (Ballarino et al., 2009; Morlando et al., 2008; Nojima et al., 2015; Pawlicki and Steitz, 2008). However, opposite to co-transcriptional splicing, which occurs progressively as the mRNA is transcribed, the co-transcriptional processing of plant pri-miRNAs would first need the transcription and folding of the entire step-loop region before it can be recognized and processed. This particularity gives a small temporal window for the processing to occur co-transcriptionally before the pri-miRNA is released to the nucleoplasm, and would likely involve some sort of RNA anchoring. Thus, it opens the question of whether the recruitment of the processing complex to the MIRNA loci induces co-transcriptional processing or only allows an earlier complex assembly. Therefore, determining whether the plant pri-miRNAs can be 
co-transcriptional processed is one of the most outstanding open questions in the field.

Co-transcriptional RNA processing is frequent in most organisms (Bentley, 2014; Herzel et al., 2017; Lee and Tarn, 2013; Peck et al., 2019; Yang et al., 2021). Such processes commonly co-exist with a post-transcriptional processing counterpart, and the balance between them can be regulated to produce alternative physiological outcomes. DNA:RNA hybrid (R-Loop) formation is also a common co-transcriptional event (Chedin, 2016; Hamperl and Cimprich, 2014). The R-loops are naturally occurring DNA-RNA hybrids formed either in cis, with the RNA encoding locus, or in trans due to sequence complementarity. Cis Rloops commonly involve the nascent transcript, especially during slow transcription (Chedin, 2016; Zatreanu et al., 2019). These chromosomal structures are frequent in bacteria, yeast, animals, and plants, playing roles in many biological processes (Crossley et al., 2019; Ginno et al., 2012; Niehrs and Luke, 2020; Santos-Pereira and Aguilera, 2015; Xu et al., 2017). In plants, Rloops play roles in development, gene regulation, and genome integrity (Ariel et al., 2020; Shafiq et al., 2017; Sun et al., 2013; Yang et al., 2017; Yang et al., 2020; Yuan et al., 2019).

Here, we used plant native elongating transcripts sequencing (plaNETseq) data to profile genome-wide nascent pri-miRNA processing intermediates associated with the RNAPII. The results indicated that pri-miRNAs are processed co-transcriptionally in Arabidopsis. This was also confirmed using different microscopic approaches of nascent pri-miRNAs. Furthermore, we found that once initiated, co-transcriptional processing can occur entirely associated with the transcriptional complex, in the case of LTB and LTBs miRNAs, or in a twostages fashion, resembling animal's pri- to pre-miRNA processing, for miRNAs processed from the base. We also discovered that co-transcriptional and posttranscriptional processing co-exist and fluctuate between growth conditions for most pri-miRNAs. Surprisingly, we found that co-transcriptional processing of primiRNAs largely relies on R-loops between the nascent transcripts and the MIRNA encoding loci to stabilize the precursor and allows DCL1 processing. Finally, our data suggest that regulation of R-loops formation directly impacts on whether a pri-miRNA is processed co-transcriptionally. 
Overall, our study identified an alternative miRNA biogenesis pathway, discovered an unexpected function for R-loops promoting RNA processing, and opened the doors to neo-functionalization of co-transcriptionally processed miRNAs with the concomitant regulatory implications.

\section{RESULTS}

\section{Imaging of pri-miRNAs suggest co-transcriptional miRNA}

\section{biogenesis.}

Current knowledge regarding the assembly of the miRNA-biogenesis complex suggests that it is possible that the processing of miRNAs is linked to transcription. To investigate whether miRNA-biogenesis and transcription are coupled, we first used Fluorescence in situ Hybridization (FISH) to visualize primiRNAs within the nucleus using confocal microscopy. For these experiments, we selected pri-miR163 and pri-miR156a as both contain introns that allow us to differentiate nascent pri-miRNAs from the mature molecules (Figure S1A). We designed the FISH probes to target an intron or exon located downstream of the stem-loop (probes named Intron and Exon, respectively), the spliced pri-miRNA transcript (Exon/Exon), the loop region (Loop), the mature miRNA region (miRNA), or the miRNA complementary sequence (miRNA*) (Figure S1A). The results showed that pri-miRNA156a and pri-miRNA163 localized in one or two discrete fluorescence spots within the nucleoplasm (Figure 1A and S1B). We then validated these results using the Stellaris FISH RNA method to detect primiRNA156a in nuclei of $A$. thaliana cells. The probes were designed against the intron sequence of pri-miRNA156a and labelled with either Quasar 570 or fluorescein (6-FAM). Again, these experiments showed that pri-miRNA156a accumulated in one or two well-defined nuclear spots (Figure S1C).

The localization of these pri-miRNA transcripts in one/two discrete loci may perfectly reflect the transcription sites of both copies of each gene. To confirm it, we used RNA Stellaris probes to analyzed subnuclear localization of primiRNA156a side by side with RNAPII immunodetection, using antibodies specific to the C-terminal domain (CTD) serine 5 and serine 2 isoforms (RNAPIISer5, RNAPIISer2). We also apply the 5-BrU incorporation method (Niedojadlo et al., 2012) to visualize newly formed transcripts. Our results showed that pri- 
miRNA156a co-localized with both transcriptionally active RNAPII and 5-BrU (Figure 1B). These results confirm that the detected spots are the pri-miRNAs transcription sites. Interestingly, we did not detect additional spots, even when using probes that could detect post-transcriptionally processed pri-miRNAs. Such distribution was also observed in animal cells by different microscopy approaches (Salataj et al., 2019; Turunen et al., 2019). This could probably represent either a very quick nucleoplasmic processing of pri-miRNAs or a diffused processing, which in turn challenges the role of D-Bodies during miRNA processing. These results encouraged us to test whether pri-miRNAs also co-localize with the miRNA biogenesis complex. Thus, we visualized pri-miRNA156a using RNA Stellaris probes followed by immunolocalization of microprocessor proteins HYL1 and DCL1. We observed pri-miR156a in one or two discrete foci while both HYL1 and DCL1 localized either dispersed in the nucleoplasm or in well-defined nuclear bodies, the so-called D-Bodies (Figure 1C and S1D). This dual distribution of HYL1 and DCL1 coincides with previous reports (Song et al., 2007). We found both proteins predominantly distributed in the nucleoplasm $(\sim 70 \%$ of all tested cells), whereas DCL1- or HYL1-containing nuclear bodies were observed in roughly $30 \%$ of cells (Figure S1D). Coincident with the reports locating DCL1 and HYL1 in MIRNA loci (Bhat et al., 2020; Cambiagno et al., 2021; Fang et al., 2015), our results showed that these two proteins co-localized with pri-miR156a transcript but not into the D-Bodies (Figure 1C, and S1E). We repeated these experiments including pri-miR163, and adjusting the stringency and acquisition parameters to focus only on well-defined structures (D-Bodies and transcription sites). Again, we observed that both pri-miRNAs transcription sites did not match D-Bodies (Figure 1D).

Altogether, our microscopy results support the idea of co-transcriptional miRNA processing, as the complex is assembled on pri-miRNA transcription sites. They also indicated that the processing complex contained in D-Bodies is not associated with the transcriptional complex. Whether these nuclear structures represent post-transcriptional pri-miRNA processing places or they are simply reservoirs of inactive proteins remain to be addressed. The relatively low number of cells displaying D-Bodies may also play against an active function in miRNA biogenesis. To further explore this aspect, we treated cells with $\alpha$-amanitin to inhibit RNAPII activity and we repeated the pri-miRNA FISH and HYL1/DCL1 
immunostaining. As expected, we did not register any fluorescence signal of primiRNA156a in plants treated with a-amanitin, confirming that transcription was successfully blocked (Figure 1E). Notoriously, we observed a shift in HYL and DCL1 subnuclear localization upon RNAPII inhibition by a-amanitin towards accumulation in nuclear bodies (Figure 1F). We confirmed this observation in planta by analyzing HYL1-YFP distribution in roots of 10-day-old $A$. thaliana plants treated with a-amanitin. After 2 hours of incubation, we detected changes in the localization of the protein toward nuclear bodies containing HYL1-YFP (Figure $1 \mathrm{G}$ and S1F). In contrast, we did not observe any changes in the distribution of two other fluorescent fusion proteins, MTA-GFP and mCherryAGO1, used as controls after $\alpha$-amanitin treatment (Figure 1G). These results indicate that RNAPII inhibition increased the number of root meristem cells containing D-Bodies, supporting a scenario where D-Bodies are not the primary source of miRNA processing acting perhaps as reservoir of miRNA biogenesis proteins.

\section{Plant pri-miRNAs are processed co-transcriptionally}

The numerous reports describing the association of miRNA biogenesis factors with pri-miRNAs encoding loci and our microscopy data prompted us to test whether such recruitment triggers co-transcriptional processing of miRNAs. To test this hypothesis, we first immunoprecipitated (IP) nascent transcripts using an antibody against the RNAPII (RIP) followed by the detection of processing intermediates by modified 5'-RACE. Plants expressing a HIS-tagged version of the ATHB1 transcription factor (Miguel et al., 2020) and an anti-HIS antibody were used as a negative control for RIP-5'-RACE. We detected processed fragments corresponding to the reported DCL1-mediated cleavage site associated with the RNAPII, but not to AtHB1, for the three tested pri-miRNAs (Figure 2A, S2A). For the pri-miR156a, where processing proceeds from the loop to the base, we detected both processing intermediates associated with the RNAPII (Figure 2A, $\mathrm{S} 2 \mathrm{~A}$ ). Aiming to confirm this observation at a genome-wide scale, we used plaNET-seq data (Kindgren et al., 2020; Leng et al., 2020) to identify pri-miRNA processing intermediates produced from nascent transcripts. In plaNET-seq, a FLAG-tagged version of NRPB2, transformed into a nrpb2-1 mutant background, is immunoprecipitated and nascent transcripts associated with the RNAPII are 
detected by Illumina sequencing (Kindgren et al., 2020). We first aligned the processed reads, from wild-type Col-0 plants, to the MIRNA loci in the Arabidopsis genome. The 5' nucleotide (nt) of each read was then plotted to identify co-transcriptional processing intermediates. MIRNA loci were scaled from miRNA-5p to the miRNA-3p to a fixed length to visualize the general processing profile. In addition, pri-miRNAs were sorted depending on their processing direction (BTL, LTB and their sequentially processed counterparts BTLs and LTBs), (Moro et al., 2018). Co-transcriptional processing intermediates, visualized as a peak in the nucleotide right after the known DCL1 cleavage sites, were easily detected, showing a clear global pattern (Figure 2B, cyan arrow). PrimiRNA transcription start sites (TSS) were observed as a region rich in undefined peaks, as expected from the variable-length from TSS to the scaled region for each individual pri-miRNA (Figure 2B, orange mark). We observed a similar narrow peak when we plotted the data to the un-scaled miRNA-3p region but not the miRNA-5p region, as expected from the processing direction (Figure $2 \mathrm{C}$ ).

Interestingly, when we plotted the 3'-nt of the plaNET-seq mapped reads, but not from the negative control, we detected the processing intermediates with even cleaner profiles (Figure 2D, S2B, S2C). The detection of such peaks suggested the retention of the 5'-end of pri-miRNAs in the transcriptional complex after processing. Such a scenario is observed in co-transcriptionally spliced mRNAs where processed exons remain bound to the complex until the splicing finish (Nojima et al., 2015). Considering that analyzing the 3'-nt end of the mapped reads eliminates the noise of TSSs and fragmented molecules, and provides cleaner profiles, we used this approach from now on. To further confirm this result, we performed a RIP experiment using an $\mathrm{H} 3$ antibody to evaluate primiRNA processing fragments associated with the chromatin. Confirming the previous observation, we found both the 3'- and 5'-ends pri-miRNAs processingfragments associated with the chromatin, but a relative depletion of the stem-loop processed regions (Figure $2 \mathrm{E}$ ). Such reduction of the hairpin region, which is not evident in the processing-defective mutants hyl1-2, is compatible with cotranscriptional processing of the pri-miRNAs (Figure 2E). The surprising retention of the 5' processed region of the pri-miRNAs in the transcriptional complex will be tackle later in this article. 
When plotting the 3'-nt of co-transcriptional processed fragments of BTL pri-miRNAs we found a clear and narrow peak coincident with the nt right before the DCL1 cleavage site (Figure 2D, 2F, S2B, and S2D; cyan arrows). Interestingly, we also detected a peak matching the last nucleotide of the miRNA$3 p$ sequence (Figure 2D, 2F, S2B, and S2D, orange arrows). The analysis of the reads ending in this nucleotide indicates that the processed hairpin (the so-called pre-miRNA) is retained temporally in the co-transcriptional processing complex. Its lower detection agrees with our RIP validations, showing a reduced association of this region with the transcriptional complex after processing (Figure $2 \mathrm{E})$. Altogether, our results indicate that plant miRNAs can be processed cotranscriptionally while nascent pri-miRNAs are still associated with the RNAPII.

\section{Processing of BTL pri-miRNAs initiates co-transcriptionally but ends}

\section{in the nucleoplasm.}

Aiming to explore whether all different biogenesis modes (LTB, LTBs, BTL and BTLs) occurs co-transcriptionally, we sorted miRNAs by their processing direction (following the annotation by (Moro et al., 2018)) and analyzed each group individually by plaNET-seq profiling.

When we analyzed LTB pri-miRNAs, two clear peaks corresponding to the first and second DCL1 cuts were detected (Figure 3A and S3A, Cyan and purple arrows). The detection of both processing intermediates indicates that the entire miRNA biogenesis occurs co-transcriptionally when the pri-miRNA is still attached to the locus. Supporting this idea, a very similar profile was observed when LTBs pri-miRNAs were analyzed (Figure 3B, and S3B). The mapping revealed a clear pattern of peaks matching each DCL1 processing site for LTBs loci (Figure 3B, and S3C). Interestingly, an additional peak corresponding to the 3 ' last nt of the miRNA-3p was visible in the global profile of both LTB and LTBs pri-miRNAs (Figure 3A, 3B, S3A and S3B, orange arrows). In LTB pri-miRNAs, this peak was only contributed by pri-miR156b, miR156e and miR408, while for LTBs, only miR159a and b contained such additional peak (Figure 3C, 3D, S3C, and S3D). Processing intermediates ending in this nucleotide (pre-miRNAs hairpin) observed before for BTL pri-miRNAs were not expected for LTB primiRNAs as the first processing step would prevent their existence. When we mapped the entire plaNET-seq reads over these loci, we found that all reads 
ending in this precise position correspond to the mature miRNA-3p (Figure 3E and $F$ ). These results show that all processing steps in LTB and LTBs pri-miRNAs occurs co-transcriptionally and that some mature miRNAs are temporally retained at their encoding loci, probably bound to a biogenesis protein such as HYL1 (Figure 3I, (Baranauske et al., 2015; Yang et al., 2010)). The observation that only some mature miRNAs derived from LTB or LTBs pri-miRNAs remain associated to their loci may represent differential affinity of each miRNA for the proteins of the biogenesis complex. It is also possible that a more efficient processing of some pri-miRNAs releases the mature miRNAs faster.

When BTL and BTLs pri-miRNAs were analyzed, we observed a peak matching the nucleotide right before the first DCL1 cleavage site but not the subsequent sites (Figure 2D, 3G, S2B, and S3E). These profiles support a scenario where only the first processing step of BTL and BTLs pri-miRNAs occurs co-transcriptionally (Figure $3 \mathrm{I}$ ). In agreement with this hypothesis, the analysis of individual pri-miRNAs revealed the partial retention of the entire processed hairpin, the so-called pre-miRNAs (Figure 2D and 3G, orange arrows), but never the mature miRNAs as it was observed for LTB and LTBs pri-miRNAs (Figure 3AE). These results indicate that BTL and BTLs pri-miRNAs suffer a first cotranscriptional processing step, but the resulting pre-miRNA is further processed in the nucleoplasm (Figure 3I). To confirm this hypothesis, we purified nucleoplasm and chromatin and quantified by RT-qPCR the abundance of the hairpin region relative to the unprocessed pri-miRNA in each fraction for LTB and BTL pri-miRNAs. Confirming our previous observation, we found that the hairpin regions of $B T L$, but not LTB, pri-miRNAs were enriched in the nucleoplasm compared to the chromatin (Figure $3 \mathrm{H}$ ). This result supports a scenario where BTL/BTLs pri-miRNAs are processed stepwise from pri- to pre-miRNA cotranscriptionally and from pre-miRNA to miRNA duplex, post-transcriptionally. An additional peak was detected downstream the 3'-nt of the miRNA-3p (Figure 3G, black arrow) corresponding to the donor site of the first exon of an AT1G12290 splicing isoform, which also contains the miR472 encoding sequence (Figure S3F). In all analyzed cases mock-IP plaNET-seq sample (negative control) showed no peaks corresponding to processing intermediates supporting that the detected signal corresponds to processed nascent RNAPII pri-miRNAs (Figure S3G). In addition, the analyzed datasets allowed us discover that miR161.1 and 
miR161.2 are unique cases of dual LTB and BTL processing from the same precursor (Figure S3H and S3I). We also used the plaNETseq data to score the processing direction of previously undefined miRNAs (Moro et al., 2018). We defined miR157d as LTB, miR2111b as BTL with retention of the mature miRNA, and miR846 as LTBs (Figure S3J). These results indicate that it is possible to use plaNET-seq to identified processing mechanisms of pri-miRNAs in different plant species, growth conditions, or mutant backgrounds.

\section{Most pri-miRNAs are processed both co-transcriptionally and post- transcriptionally.}

The detection of co-transcriptional processing does not necessarily imply that all miRNAs, or even not all pri-miRNAs transcripts from a single locus, are processed exclusively during transcription. Full-length pri-miRNAs can be found in the cells and even move to the cytoplasm to translate into small peptides (Lauressergues et al., 2015). Thus, some pri-miRNAs, or at least a fraction of the transcripts from each locus, escape co-transcriptional processing. This is a scenario similar to splicing, where both co-transcriptional and post-transcriptional mRNA processing co-exist. To evaluate the extent of co-transcriptional processing, we re-analyzed plaNET-seq data and calculated the ratio between those reads ending at DCL1 cleavage site (co-transcriptionally processed, Figure $4 \mathrm{~A}$, green lines) and those expanding the site (unprocessed pri-miRNAs, Figure $4 \mathrm{~A}$, blue and cyan lines). Although, unprocessed pri-miRNAs associated with the chromatin will either be processed in the nucleoplasm or exit the nucleus unprocessed, we will refer to these molecules as post-transcriptionally processed pri-miRNAs. In the case of BTL or BTLs pri-miRNAs, we did this calculation using the signature peak at the 5'-end of the transcript (Figure 4A, site "a"), since the subsequent cuts are undetected and happen post-transcriptionally, as we showed before. Conversely, we used the first cleavage site toward the loop region for LTB and LTBs (Figure 4A, site "a"), as the following sites overestimate the unprocessed reads by counting processing intermediates (cyan lines). To simplify the analysis, we excluded those MIRNA loci without plaNET-seq mapped reads. We used two independent experiments and plotted the co-transcriptional vs posttranscriptional processing ratio for each pri-miRNA sorted by processing type 
(Figure 4B). In the tested conditions, and among the pri-miRNAs showing both processing components, the analysis revealed that $\sim 25 \%$ of the pri-miRNAs were preferentially co-transcriptionally processed, with miR166b miR165a, miR393a, miR162a, and miR160b standing out as primarily processed co-transcriptionally (Figure 4B-D). Approximately $8 \%$ of analyzed miRNAs showed an equal preference for co-transcriptional and post-transcriptional miRNA processing (Figure 4B-D). While $\sim 67 \%$ of pri-miRNAs appeared to be processed more posttranscriptionally (Figure 4B-D). From those, it is worth noting that $\sim 31 \%(21 \%$ among all) have a ratio $>$ to 0.5 , indicating that those miRNAs are processed cotranscriptionally more than a quarter of the time (Figure 4B-D). Several primiRNAs, including those encoding miR862, miR399b, miR165b, and miR394b, stand out among those with a nearly undetectable signal of co-transcriptional processing (Figure 4B). These results suggest that pri-miRNAs co-transcriptional processing is likely a dynamic and potentially regulated process, not identical for each locus.

\section{Co-transcriptional miRNA processing efficiency and frequency} fluctuate depending on the conditions.

We next wondered whether the transcriptional activity of the RNAPII or even the environment could affect the balance between co-transcriptional and post-transcriptional processing of pri-miRNAs. We first repeated our previous analyses using plaNET-seq data obtained from plants incubated for 12 hours at $4{ }^{\circ} \mathrm{C}$. The results indicated that reducing the plant growing temperature impact the ratio between co-transcriptional vs post-transcriptional processing (Figure $5 A$ ), especially for some individual miRNAs (Figure S4A, S4B). Among all tested pri-miRNAs, a comparison of the co-/post-transcriptional processing ratios between samples (with a threshold of $+/-50 \%$ difference) underlines a considerable number of pri-miRNAs affected by this condition either in a negative or positive way (Figure S4B).

The ratio of exon/intron inclusion, one of the best-studied co-transcriptional RNA maturation processes, is well known to depend on RNAPII elongation speed (Dujardin et al., 2014; Giono and Kornblihtt, 2020; Godoy Herz and Kornblihtt, 2019). Thus, we wondered whether the pri-miRNAs co-transcriptional/posttranscriptional ratio is also affected by the RNAPII activity. To test this possibility, 
we analyzed plaNET-seq data obtained from nrpb2-1 plants complemented with a wild type NRPB2:FLAG construct $\left(N R P B 2^{\mathrm{wt}}\right)$ or with a version of this protein containing a Y732F mutation (NRPB2 ${ }^{\mathrm{Y} 732 \mathrm{~F}}$ ) which accelerates RNAPII transcription in vivo (Leng et al., 2020). Using a difference ratio threshold of 0.5, we found that, with a few exceptions, an increase in RNAPII speed produces a reduction in co-transcriptional processing. However, this is evident for a relatively small fraction ( $22 \%$ ) of all analyzed miRNAs (Figure 5B, S4C, and S4D). Given that at least the entire stem-loop region of a pri-miRNA needs to be transcribed before processing starts, our result may imply that a quick transcription releases the mature pri-miRNA transcript before it is co-transcriptional processed.

Next, we analyzed the co-transcriptional processing profile in NRPB2 ${ }^{\mathrm{Y} 732 \mathrm{~F}}$ plants, asking whether RNAPII speed may affect co-transcriptional processing efficiency rather than frequency. Overall, we did not find any noticeable effect over pri-miRNAs processed BTL beyond an increment in the retention of the hairpin (Figure 5C). Interestingly, we found an apparent enhanced processing efficiency in LTB and LTBs pri-miRNAs (Figure 5C). In particular, it was interesting to observe that such increase in the processing efficiency was progressive from the initial DCL1 cut and become more evident in the subsequent cuts. This intriguing result may represent partial, alternative, or misfolded primiRNAs generated depending on the polymerization speed. Although, the number of LTBs pri-miRNAs is small and we cannot discard this as a coincidence.

Overall, these results suggest that the balance between co-transcriptional and post-transcriptional processing of each pri-miRNA is dynamic and responds to specific conditions. This opens the question of whether a miRNA may have different functions depending on when/where it is processed. Recently, we have reported that HST associates with MIRNA loci through the interaction with the mediator complex, recruiting DCL1 to the MIRNA loci (Cambiagno et al., 2021). Thus, we reasoned that HST mutants might have an imbalance of the processing ratio that can help us study the dynamics of co-/post-transcriptional processing. We measured the amount of processed and un-processed pri-miRNAs in the nucleoplasm and chromatin of Col-0 and hst-15 mutant plants to test this hypothesis. Confirming hst-15 as a model of impaired co-transcriptional processing, we found a reduction in the ratio of processed/non-processed nascent pri-miRNAs in the IP fraction in the mutants compared to WT plants 
(Figure 5D). We observed the opposite pattern in the nucleoplasm fraction, suggesting that the balance between co-transcriptional and post-transcriptional processing swoop in this mutant, but the overall miRNA production is compensated (Figure 5E). Coincidentally, small RNA sequencing analysis of $h s t-$ 15 mutants revealed that most miRNAs, and particularly those with high co-/posttranscriptional ratio, are not altered in the mutants as previously reported (Brioudes et al., 2021), supporting a change in processing type rather than an overall effect on miRNA biogenesis (Figure 5F). Recently, it was shown that HST mutants display a compromised non-cell-autonomous miRNA function cause by an impaired movement of mature miRNAs (Brioudes et al., 2021). Notoriously, most miRNAs reported to act non-cell-autonomously, such as miR160, miR165, and miR166 (Brioudes et al., 2021; Brosnan et al., 2019; Fan et al., 2021), ranked among the highest co-transcriptionally processed miRNAs in our data (Figure 4B). In agreement with the reports suggesting that AGO1-unloaded miRNAs are likely the mobile component (Brioudes et al., 2021; Devers et al., 2020; Fan et al., 2021), highly co-transcriptionally processed miRNAs appeared among the less efficiently loaded in AGO1 in RIP-seq experiments (Figure 5G). This suggests that co-transcriptionally processed miRNA may undergo a different fate after processing. This idea goes in line with the reduced co-transcriptional miRNA processing observed in hst-15 (Figure 5D) and the lack on miRNA movement, without a change in the mature miRNA steady levels, previously reported for this mutant (Brioudes et al., 2021).

\section{R-Loops formation between pri-miRNAs and the encoding loci} promote co-transcriptional miRNA processing.

One of the most intriguing results so far was the systematic detection of the processed $5^{\prime}$ region of the pri-miRNAs in the NRPB2-IP fraction. Similar profiles were previously reported, for example, during splicing due to the stabilization of the processed mRNA fragments by the spliceosome complex (Nojima et al., 2015). Different from splicing, where each intron can be removed while transcription proceeds, co-transcriptional pri-miRNA processing required the transcription of at least the entire stem-loop region before the processing complex can recognize the features necessary for DCL1-mediated miRNA biogenesis. Thus, it is possible that the observed retention of the 5' ssRNA arm 
in the IP samples represent pri-miRNA transcripts either stabilized or anchored to the loci to become co-transcriptionally processed. The observation in our microscopy assays that spliced pri-miRNAs are still associated to their transcription sites (Figure 1) also support a scenario were pri-miRNAs are temporally anchored to the encoding loci. The apparent effect of transcriptional speed on the co-processing efficiency made us wonder, as well, whether transcription influences such anchoring. A slow transcription may cause, for example, the formation of R-Loops between an RNA and its encoding locus, which in turn could lock the transcript in the locus (Zatreanu et al., 2019). Within such a scenario, an R-loop that is formed between a MIRNA encoding locus and the nascent transcript may stabilize and anchor the pri-miRNA to allow cotranscriptional processing once the transcription and folding of the dsRNA hairpin structure end. We explored this possibility by analyzing DRIP-sequencing (DRIPseq) data (Xu et al., 2020; Xu et al., 2017), searching for the presence and pattern of R-Loop formation over MIRNA loci. To do this analysis, we first scaled all primiRNAs using either a fixed window from the transcription start site (TSS) to the most up-stream DCL1 cleavage site (Figure 6A, green line) or from this site to the end of miRNA-3p (Figure 6A, orange line). To establish such windows, we first defined each pri-miRNA TSS as described in Materials and Methods. Then, we scored the R-Loop signature for each pri-miRNA, and plotted the metagene profile of R-loops over MIRNA loci (Figure 6B). We found frequent R-loops over the analyzed loci in the DRIP-seq dataset, an observation confirmed by DRIPqPCR on some specific loci (Figure 6B, and 6C). However, it was clear that the pattern of R-loop formation was variable among tested pri-miRNAs. Thus, we sorted all pri-miRNAs into categories depending on their R-Loop profile (Figure 6D). We observed five types of MIRNA genes: those not showing R-loops at all (Figure 6D $(\alpha)$ ), those with R-loops restricted to the 5'-ssRNA arms of the primiRNAs (Figure 6D $(\beta)$ ), those with an R-loop like $\beta$ but with an additional signature toward the end of the loci (Figure 6D $(\gamma)$ ), those where the R-Loops extend uniformly over the entire loci (Figure 6D $(\delta)$ ) and finally miRNAs with colliding R-loops, probably a reflection of bi-directional transcription (Figure 6D $(\varepsilon)$ ). Strikingly, when we asked whether the presence of an R-Loop impacts cotranscriptional processing, measured as the ratio co-/post-transcriptional 
processing, we found that miRNAs with R-Loops in the initial single stranded region of the transcripts ( $\beta$ and $\gamma$ signatures) are preferentially processed cotranscriptionally (Figure 6E). Conversely, pri-miRNAs not displaying R-loops over the loci or the characteristic R-Loop near the TSS ( $\alpha$ or $\delta, \varepsilon$ respectively) showed poor signs of co-transcriptional processing (Figure 6E).

Remarkably, when we analyzed polycistronic miRNA duets encoded by a single transcript, we observed that the miRNA hairpin located adjacent to an Rloop was processed co-transcriptionally more efficiently or even the only one showing signs of co-transcriptional processing (Figure 6F). This goes in line with a recent report indicating that polycistronic $5^{\prime}$ hairpins are processed more efficiently than 3' hairpins (Lunardon et al., 2021). It also reinforces the hypothesis that R-loops between nascent pri-miRNAs and its encoding loci anchor and stabilize the molecule allowing the co-transcriptional processing. Interestingly, in the case of MIR842/MIR846 duet, the second miRNA, but not the first, shows signs of co-transcriptional processing (Figure 6F). This is coincident with an extended R-loop encompassing the entire region encoding MIR842, likely impairing its processing as observed for class $\delta$ MIRNAs, but stabilizing the second hairpin to allow miR846 co-transcriptional processing as $\beta$ MIRNAs.

When we explored some miRNAs encoded within protein-coding genes, we found that miR402, which is processed form a short transcript (Knop et al., 2017) and encoded in the first intron of the hosting gene near the TSS and associated with an R-Loop, is efficiently processed in a co-transcriptional way (Figure 6G). Conversely, miRNAs encoded away from the TSS and the associated R-loops, such as miR156g, miR862 or miR838, do not show cotranscriptional processing signs or only an inefficient processing (Figure 6G). This observation reinforces the idea that R-loops in the proximity and upstream of the hairpin promotes co-transcriptional processing of pri-miRNAs.

Interestingly, parallel studies using similar, but not identical, samples (Xu et al., 2020; Xu et al., 2017) showed different R-loop patterns in some miRNA loci (Figure S5). This suggest that R-loop formation may change depending on the plant developmental stage or growth condition and potentially regulate cotranscriptional miRNA processing. We mined DRIP-seq data (Xu et al., 2020) searching for growth conditions with altered R-loop formation patterns to explore 
further whether R-loops initiate and regulate co-transcriptional processing. We focused only on those MIRNA loci with significant changes in the processing ratio (Figure 5). An alteration in the R-loop patterns may represent a regulatory mechanism that translates into changes in the processing mechanisms and potentially on the mature miRNA fate. Among the tested condition, we found that prolonged heat stress (30 hours at $37^{\circ} \mathrm{C}$ ), either control or after a period of recovery at $23{ }^{\circ} \mathrm{C}$, produced consistent changes in the R-Loop patterns over MIR160a, MIR160b, MIR165a, MIR166a, and MIR166b among others (Figure $6 \mathrm{H})$. In agreement with the role of R-loop in promoting co-transcriptional processing, we observed that the same temperature treatment also translated in a reduction of the co-transcriptional processing ratio measured as an increment in unprocessed pri-miRNAs in RIP-qPCR samples (Figure 6I, left panel). This change in co-transcriptional processing was not observed for pri-miR164b, which does not present an R-loop over its locus and showed undetectable signs of cotranscriptional processing (Figure 6I, left panel). In addition, we also found that the processed ssRNA arm of pri-miR165a, 166a, and 166b, but not 164b used as a negative control, were less retained in the RIP fraction of heat stressed plants coincident with the reduction in R-loops (Figure 6I, right panel). These results position the co-transcriptionally formed R-loops as a critical element to anchor and trigger co-transcriptional processing of pri-miRNAs in plants. This also suggested that the regulation of R-loops resolution directly impact how miRNAs are produced. Even when it is likely that the miRNA biogenesis complex also helps to stabilize the nascent pri-miRNAs in the transcriptional complex, the formation of R-loops appeared as a critical event toward achieving cotranscriptional miRNA processing.

\section{Discussion}

The coupling of transcription and RNA processing is a common feature in most organisms. Nascent RNAs undergo several processing steps cotranscriptionally, including 5'-capping, splicing, polyadenylation, as well as chemical modifications such as m6A (Bentley, 2014; Lee and Tarn, 2013; Yang et al., 2021). In animals, the processing of pri-miRNAs is not an exception and is accepted to occur co-transcriptionally (Morlando et al., 2008; Nojima et al., 2015; Pawlicki and Steitz, 2008). However, it was unclear whether this process also 
occurs co-transcriptionally in plants despite many miRNA-biogenesis factors associate with MIRNA loci. The long and structurally variable plant pri-miRNAs posed a challenge for such an event to happens. On top, the recent demonstration of pri-miRNA processing in SERRATE-containing liquid droplets, likely D-Bodies, and the absence of this protein in most MIRNA loci challenged the idea of co-transcriptional processing in plants (Speth et al., 2018; Xie et al., 2021). In this study we confirmed that pri-miRNAs are processed cotranscriptionally in plants and showed that this process co-exists with a posttranscriptional counterpart. This implies that two alternative pathways, perhaps involving a different set of proteins, co-exist and may produce miRNAs with alternative functions. We have shown that HST is required for DCL1 association to MIRNA loci and pri-miRNA co-transcriptional processing (Cambiagno et al., 2021), Figure 5F, and G). Recently, it was also demonstrated that HST is required for the non-cell-autonomous function of miRNAs (Brioudes et al., 2021). However, it is unclear the mechanism involved in such an HST-depended miRNAmovement. Based on the results presented here, it is tempting to speculate that co-transcriptionally processed miRNAs, but not their post-transcriptionally processed siblings, constitute the mobile pool of miRNAs. It is possible, for example, that AGO1 preferentially loads post-transcriptionally processed miRNAs sealing their fate as non-mobile molecules. In this scenario, the interaction of AGO1 with nucleoplasmic exclusive miRNA partners, such as CARP9 or TRANSPORTIN1 (Cui et al., 2016; Tomassi et al., 2020), but not with the chromatin-associated complex, may sort which miRNAs are loaded. On the other hand, co-transcriptionally processed miRNAs may scape nuclear AGO1loading by missing partner proteins, shuttling to the cytoplasm either free or associated with chaperon proteins, such as HYL1, and then become mobile. However, it was reported that AGO1 could also interact with the chromatin and even RNAPII (Huang et al., 2013; Liu et al., 2018) suggesting that the outcome of co-transcriptional processed miRNA may be unique for each loci. It is worth noting that miRNAs know to act non-cell-autonomously, such as miR160, miR165, and miR166, rank on top of the most co-transcriptionally processed miRNAs in our analysis (Figure 4). Interestingly, our study showed that the preference for co- or post-transcriptional processing swoop for mobile miRNAs in 
hst mutants which translate in nearly unaffected mature miRNA levels the mutants as previously reported (Brioudes et al., 2021) (Figure 5F-H).

Two proposed models explain co-transcriptional modifications of RNAs in animals and yeast. A first model, known as the recruitment model, relies on the transcriptional machinery to recruit RNA processing factors to trigger the events (Bentley, 2014). This model appears relevant during the coupling of transcription and pri-miRNA processing in plants as many miRNA biogenesis factors rely on the interaction with the RNAPII transcriptional complex to associate to MIRNA loci. In a second model, the kinetic model (de la Mata et al., 2010), the relative rates of transcription elongation directly impact RNA processing. This is the case of splicing or poly $(A)$ sites that are recognized or skipped depending on the RNAPII speed producing alternative transcript isoforms. In this process, a slow elongation rate provides RNA processing factors with more time to recognize processing sites, to assemble the complexes, and to produce the modifications (Dujardin et al., 2014). This model could also be particularly relevant for plant primiRNAs co-transcriptional processing, as the long transcript requires time, and a large percentage of transcription completion to fold correctly. It was recently shown that the transcription elongation rate impacts nascent RNA folding (Saldi et al., 2018; Scharfen and Neugebauer, 2021). Thus, a change in the elongation rate may affect the structure of pri-miRNA folding and how it is processed, as previously shown (Wang et al., 2018). Interestingly, $\mathrm{N}^{6}$-adenosine m6A methylation, a co-transcriptional RNA modification (Knuckles et al., 2017), was show to impact RNAPII pausing (Akhtar et al., 2021) and pri-miRNA processing (Bhat et al., 2020) providing another potential link between co-transcriptional events and miRNA biogenesis. In this sense, our data indicated that elongation speed affect co-transcriptional processing, perhaps by controlling R-loop formation (Figure 5). However, such a potential effect needs to be studied case by case rather than in a metagene analysis as each pri-miRNA structure is unique and affected differently.

RNAPII speed (elongation rate) is regulated in response to intra- and extracellular stimuli changing transcriptome composition in turn (Muniz et al., 2021). Interestingly, R-loops are formed during transcription and impact RNAPII elongation (Aguilera and Garcia-Muse, 2012). In turn, slow elongation by RNAPII also increases co-transcriptional R-Loop formation (Zatreanu et al., 2019). This 
implies that the stimuli the cells receive regulate R-loop formation. Here, we showed that the hybridization of the nascent pri-miRNA with their encoding loci promotes co-transcriptional processing by anchoring the 5' ssRNA arm of the primiRNA to the loci by R-loop formation. R-loops have previously been linked to many biological processes by directly affecting transcription and genome stability. In our model, it is likely that R-loops adjacent to the TSSs of MIRNA loci stabilize the nascent pri-miRNA, and provide the time required to transcribe and fold the stem-loop region, and to assemble the miRNA-biogenesis complex cotranscriptionally. To the best of our knowledge, this is the first time such a positive regulatory function is attributed to R-loops as a feature improving the RNA own processing. Nevertheless, it can be argue that R-loop formation could potentially negatively impact MIRNA transcription as RNAPII would collide with the hybrid (Aguilera and Garcia-Muse, 2012). However, it has been shown that R-loops are dynamic structures with a rapid turned over of a half-life of 10 min (Chedin, 2016; Sanz et al., 2016). Thus R-loops are continuously formed and resolved, allowing only temporal retention of nascent transcripts, probably only sufficient to process the pri-miRNA co-transcriptionally in this case.

In summary, our study provides evidence of the existence of a cotranscriptional processing pathway in plants, a mechanism that co-exists with a canonical nucleoplasmic process. We also provide new insights into mechanisms of pri-miRNA processing that depends on processing direction. We found that the co-transcriptional processing of BTL miRNA resembles the animal pathways with more defined pri-miRNA>pre-miRNA>miRNA steps. Conversely, LTB primiRNAs follows a more fluid path with continuous processing steps that blurs the canonical stages. The discovery that R-loops between the nascent pri-miRNAs and the encoding loci promotes coupling between transcription and processing provides an novel regulatory scenario that can re-define the function of a mature miRNA. Identifying the proteins, likely RNA-helicases, which help resolving these $\mathrm{R}$-loops is imperative to manipulate the ratios of co-transcriptional processing and study the potential function of the produced miRNAs.

\section{Material and methods}

\section{Plant material and growth condition}


Arabidopsis thaliana ecotype Columbia (Col-0), transgenic and mutant plants were grown at $23^{\circ} \mathrm{C}$ on plates containing $2.2 \mathrm{~g} / \mathrm{L}$ of Murashige-Skoog (MS) medium ( $\mathrm{pH} \mathrm{5.7)} \mathrm{and} \mathrm{0.6 \%} \mathrm{agar} \mathrm{in} \mathrm{long-day} \mathrm{photoperiod} \mathrm{(LD,} 16$ hours of light/8 hours of dark). Seeds were disinfected with $10 \% \mathrm{v} / \mathrm{v}$ bleach and $0.1 \%$ SDS and stratified in $0.1 \%$ agar for 3 days at $4^{\circ} \mathrm{C}$ before sowing. Arabidopsis thaliana seed ecotype Columbia (Col-0), hst-15 (SALK_079290), hyl1-2 (SALK_064863), ATHB1-HIS (Miguel et al., 2020), NRPB2-FLAG (Onodera et al., 2008), and NRPB2 ${ }^{Y 732 F}$-FLAG (Leng et al., 2020), were used in this study. For isolation chromatin/nucleoplasm RNA experiments the plants were grown for 20 days under before collecting the samples. For long heat stress (LHS) treatments the seeds were grown in $1 / 2$ - strength MS medium complemented with $0.6 \%$ agar for 12 days at $23^{\circ} \mathrm{C}$, then the seedlings were transferred to $37^{\circ} \mathrm{C}$ for $30 \mathrm{hrs}$. before returning then to $23^{\circ} \mathrm{C}$ for $12 \mathrm{~h}$. For FISH experiments the plants were grown on Jiffypots $®$ (Jiffy-7 42 mm; Jiffy Products International AS, Norway) and stratified for 2 days in dark at $4{ }^{\circ} \mathrm{C}$. The immunolabeling of $\mathrm{BrU}$ and $\alpha$-amanitin treatment experiments were performed on isolated nuclei of 2-week-old seedlings grown on $1 / 2$ - strength MS medium complemented with $0.8 \%$ agar. Before isolation, the plants were fixed in $4 \%$ paraformaldehyde in phosphate-buffered saline (PBS), $\mathrm{pH} 7.2$.

\section{Isolation chromatin/RNAPII-bound RNA and nucleoplasm RNA.}

Between 3-4 grs of plant material was frozen in nitrogen liquid and grinded in a mortar. The powder was resuspended in $30 \mathrm{~mL}$ of Extraction Buffer 1 (10mM Tris-HCl pH8; 0.4M sucrose; 10 mM MgCl $2 ; 5$ mM BME; 0.2 mM PMSF; RNasin PROMEGA) and was filter thought a Nylon membrane of $150 \mu \mathrm{m}$ and centrifuged at $2000 \mathrm{~g}$ for $20 \mathrm{~min}$ at $4^{\circ} \mathrm{C}$. The pellet was washed twice time with Extraction Buffer 2 (10mM Tris-HCl pH8; 0.25 M sucrose; 10 mM MgCl$; 5$ mM BME; $1 \%$ TRITON X100; $100 \mu \mathrm{M}$ PMSF) and centrifuged at $2000 \mathrm{~g}$ for $10 \mathrm{~min}$ at $4{ }^{\circ} \mathrm{C}$. Then we added $500 \mu \mathrm{l}$ of Extraction Buffer 3 (10mM Tris-HCl pH8; $1.7 \mathrm{M}$ sucrose; $2 \mathrm{mM}$ $\mathrm{MgCl}_{2} ; 5 \mathrm{mM} \mathrm{BME} ; 0.15 \%$ TRITON X100) to the pellet. This solution was gently placed on a $1500 \mu \mathrm{l}$ column of Extraction Buffer 3 and centrifuged at $13000 \mathrm{~g}$ for $5 \mathrm{~min}$ at $4^{\circ} \mathrm{C}$. The pellet obtained was resuspended in $500 \mu \mathrm{l}$ Lysis Buffer $(0.3 \mathrm{M}$ $\mathrm{NaCl} ; 20$ mM Tris-HCl pH7.5; $5 \mathrm{mM} \mathrm{MgCl}_{2} ; 5$ mM DTT; proteases inhibitor tablet) and incubated at $4^{\circ} \mathrm{C}$ for $2 \mathrm{~h}$ in a rotator. We took $10 \%$ of the sample and saved 
it as INPUT, 45\% for IgG-IP (AS09 605, Agrisera) negative control, and 45\% used for the RIP experiment. For this, $30 \mu$ of SureBeads (Protein A Magnetics Beads, BioRad) and 1/1000 of Histone 3 (H3 AS10 710, Agrisera) or RNAPII (AS11 1804, Agrisera) antibody were added to the sample and incubated in rotation at $4^{\circ} \mathrm{C}$ overnight. The IP fraction was saved as RIP-sample while the supernatant as nucleoplasm. The RIP fraction was washed with Washing Buffer $(0.3 \mathrm{M} \mathrm{NaCl} ; 20$ $\mathrm{mM}$ Tris-HCl pH7.5; 5 mM MgCl $2 ; 5$ mM DTT; protease inhibitor tablet; RNasin PROMEGA) three times. Treatment with Proteinase $\mathrm{K}$ was carried out in $500 \mu \mathrm{l}$ of PK Buffer (100 mM Tris-HCl pH8; 50 mM NaCl; 10 mM EDTA; Proteinase K 4 $\mathrm{mg} / \mathrm{ml}$; RNasin PROMEGA) $2 \mathrm{~h}$ at $55^{\circ} \mathrm{C}$ and $15 \mathrm{~min}$ at $95^{\circ} \mathrm{C}$. We added $2 \mathrm{U}$ of DNase I (Thermo Fisher) to the sample and incubated them for $30 \mathrm{~min}$ at $37^{\circ} \mathrm{C}$. RNA extraction was then performed with $1 \mathrm{ml}$ of TRIZOL and $200 \mu$ l of chloroform. Precipitation was done with $1 \mu \mathrm{l}$ glycogen, acetate of sodium $3 \mathrm{M} \mathrm{pH} 2.5$, and isopropanol at $-20^{\circ} \mathrm{C}$ overnight. The reverse transcription was performed with EasyScript Reverse Transcriptase (M-MLV, RNase H-, TransGen Biotech) and dN6 according to the manufacturer recommendations. Quantitative RT-qPCRs, were performed using three independent biological replicates. U6 was used as a housekeeping loading control. Averages from biological replicates and SEM were calculated from $2^{-\Delta \Delta C t}$ values, and the error displayed as two times SEM. Each replicate was treated as independent samples for statistical analysis. Statistical differences between samples were determined by an unpaired, two-tailed, t-test analysis. See supplementary table $\mathrm{S} 1$ for oligonucleotide primers.

\section{DNA-RNA Immunoprecipitation (DRIP).}

This experiment was performed with 3 grams of plant material previously frozen in nitrogen liquid. After grinded of samples, we performed the nucleus purification as described above. The chromatin pellet was resuspended in $300 \mu \mathrm{l}$ Nuclei Lysis Buffer (50 mM Tris-HCl pH8; 0.1 \% SDS; 10 mM EDTA). We added $300 \mu$ l of Proteinase K Buffer 2X (200 mM Tris-HCl pH7.5; 100 mM NaCl; 20 mM EDTA; RNasin PROMEGA $20 \mathrm{U} / \mu \mathrm{l} ; 0.04 \mathrm{mg} / \mathrm{ml}$ Proteinase $\mathrm{K}$ ) and incubated for $1 \mathrm{~h}$ at $55^{\circ} \mathrm{C}$. We then added 1 volume of Phenol-Chloroform-Isoamyl acid $(25: 24: 1)$ solution, mixed and centrifuged for $15 \mathrm{~min}$ at $12000 \mathrm{rpm}$ at $4^{\circ} \mathrm{C}$. We took the upper phase and transferred it to a new tube. We added 1 volume of 
chloroform, mixed and centrifuged for $15 \mathrm{~min}$ at $12000 \mathrm{rpm}, 4^{\circ} \mathrm{C}$. We sonicated the upper phase in Refrigerated PicoRuptor for 4 cycles, 30 seconds $\mathrm{ON}-30$ seconds OFF. In this step, the sample was divided into three fractions: $10 \%$ of the sample was used as an INPUT, $45 \%$ for RNase $\mathrm{H}$ treatment as negative control, and $45 \%$ for DNA-RNA immunoprecipitation with 59.6 antibody (MABE1095 Millipore-SIGMA) and Dynabeads Protein G overnight at $4{ }^{\circ} \mathrm{C}$. The next day, the beads were washed three times with ChIP Dilution Buffer $(1.1 \%$ TRITON X100; 1.2 mM EDTA; 16.7 mM Tris-HCl pH8; $167 \mathrm{mM} \mathrm{NaCl)} 5$ min in rotation at $4^{\circ} \mathrm{C}$. After the washes we resuspended the beads in $500 \mu \mathrm{l}$ of Proteinase $\mathrm{K}$ Buffer $1 \mathrm{X}$ and incubated at $55-65^{\circ} \mathrm{C}$ for $1 \mathrm{hr}$ and at $95^{\circ} \mathrm{C}$ for $15 \mathrm{~min}$. The DNA-RNA purification was performed with Phenol-Chloroform-Isoamyl. After washing with chloroform, we added $1 \mu$ of glycogen, $10 \%$ volume of $\mathrm{NaAc} \mathrm{pH} 5.2$, and two-volume of absolute ethanol and incubated it overnight at $-20^{\circ} \mathrm{C}$. DNARNA was recovered by centrifugation for $30 \mathrm{~min}$ at $12000 \mathrm{rpm}, 4^{\circ} \mathrm{C}$. The pellet was washed with $300 \mu$ of ethanol $70 \%$. The dry pellet was resuspended with 30 $\mu \mathrm{l}$ of water supplemented with $0.5 \mu$ of RNAse A (EN0531 Thermo-Fisher) and the resulting DNA used for $\mathrm{qPCR}$ analysis as described before.

\section{RNA analysis.}

The rapid amplification of $5^{\prime}$ cDNA ends (5' RACE) method to detect processing intermediates was carried out from RNAPII-IPed samples as follow: First a nuclei isolation was performed as previously described. Purified nucleus were recovered in $300 \mu \mathrm{l}$ of Nuclei Lysis Buffer (50 mM Tris-HCl pH8; 0.1\% SDS; 10 mM EDTA; 100 HM PMSF; RNasin PROMEGA) and 5 cycles (30 s ON - $30 \mathrm{~s}$ OFF) of cell disruption applied with a refrigerated PicoRuptor. The samples were centrifuged for $10 \mathrm{~min}$ at $13000 \mathrm{~g}, 4^{\circ} \mathrm{C}$ and the supernatants were incubated overnight with 1/100 RNAPII or HIS antibodies (RNAP II AS11 1804, HIS AS20 4441, Agrisera) and $30 \mu$ SureBeads (BioRad). Three washes were performed with ChIP Dilution Buffer as in DRIP assays before incubating the IP fraction with Proteinase $\mathrm{K}$ for $2 \mathrm{~h}$ at $55^{\circ} \mathrm{C}$ plus $15 \mathrm{~min}$ at $95^{\circ} \mathrm{C}$ in PK Buffer $(100 \mathrm{mM}$ Tris- $\mathrm{HCl}$ pH8; 50 mM NaCl; 10 mM EDTA; 4 mg/ml Proteinase K; RNasin PROMEGA). We added $2 \mathrm{U}$ DNase I (ThermoFisher) and incubated it for $30 \mathrm{~min}$ at $37^{\circ} \mathrm{C}$. After that, we continued with RNA purification as we describer before. 
The 5' RACE method was performed as as described previously (Llave et al., 2002). In brief, $2 \mu$ of IP-RNA, or input RNA, were ligated to a RNA adapter (GeneRacer ${ }^{\mathrm{TM}}$ RNA, Thermofisher). Next, we used random primers for first strand cDNA synthesis. PCRs were performed with pri-miRNA specific reverse primers (Table S2). The amplification products were purified, cloned in pGEMTeasy vectors, and sequenced.

Small RNA sequencing of hst-15 mutants was previously described (Cambiagno et al., 2021). Previously described AGO1-associated miRNAs datasets (Mi et al., 2008) were used to estimate loading of each miRNA. For this, we first calculated the fraction each miRNA represents to the total number of read in the input and IP samples. Then a ratio between the fractions in the IP/input was calculated to estimate the loading preference of each miRNA.

\section{Bioinformatics analysis}

Chromosomal coordinates of Arabidopsis thaliana miRNAs were downloaded from the miRbase sequence database v22 (Kozomara et al., 2019). Hairpin precursor coordinates (annotated as "miRNA primary transcript", pri-miRNA) and mature miRNAs coordinates were sorted by their biogenesis direction as indicated by (Moro et al., 2018) and each group was analyzed separately. The pri-miRNAs were scaled in order to exclude 5' and 3' arms to avoid noise signals when profiling plaNET-seq data. BTL, LTB, and LTBs pri-miRNAs were scaled from the miRNA-5p to the miRNA-3p chromosomal coordinates, and BTLs primiRNAs from the first DCL1 cut to the miRNA-3p genomic coordinates. Custom $R$ scripts were written for this purpose and annotation files were manually curated. For the analysis of R-Loops formation over MIRNA loci, two coordinates windows were defined as follows: one from the TSS to the most up-stream DCL1 cleavage site, and the other from this site to the end of miRNA-3p. To define the first window, each pri-miRNA TSS was annotated de novo by combining information from different sources: PTSmiRNA database (You et al., 2017), TSS of Arabidopsis MIRNA primary transcripts reported by (Xie et al., 2005), mapped plaNET-seq reads (Kindgren et al., 2020), Arabidopsis thaliana ESTs and Full lenght cDNAs (Campbell et al., 2014), paired-end analysis of transcription start sites (Morton et al., 2014) and genome-wide TSS sequencing (Nielsen et al., 2019). 
Samples from selected sequencing studies (Table S2) were downloaded from public repositories in bigWig format. The ssDRIP-seq mapped reads are available as forward (wR-loops, representing an R-loop formation containing ssDNA on the Watson strand and an DNA:RNA hybrid on the Crick strand) and reverse reads (cR-loops; (Xu et al., 2017)). In addition, reads from selected samples of plaNETseq experiments were downloaded in SRA format and converted to fastq format using fasterq_dump (SRA-Toolkit, https://trace.ncbi.nlm.nih.gov/Traces/sra/sra.cgi?view=software).

Trimming, alignment to TAIR10 genome and post-processing of plaNET-seq reads were done as previously described (Kindgren et al., 2020; Leng et al., 2020), using the 01-Alignment_plaNET-Seq.sh and 02-Postprocessing_plaNETSeq.R scripts available in the code repository: https://github.com/MaximIvanov/Kindgren et al 2019. The script loadNETSeqBAM.R was modified in line 65 to obtain the genomic coverage in the 5' nucleotide of mapped reads (mode $=$ "start") or the full coverage (mode = "whole_read"). In each case, genomic coverage was exported as strand-specific bigWig and bedGraph files using rtracklayer_1.42.2.

For the preparation of metagene plots of plaNET-seq data, the two biological replicates of each sample were merged using the bigWigMergePlus tool (https://github.com/c3g/kent/releases/tag/bigWigMergePlus 2.0.0). For some plots, strand-specific files were shown separately. The deepTools suite (Ramirez et al., 2016) was employed to draw metagene plots of plaNET-seq and SsDRIPseq samples. ComputeMatrix tool was used in the scale-regions mode followed by plotProfile tool (parameters used are described in Table S3).

To calculate the ratio of co-transcriotional vs. post-transcriptinal processing each individual pri-miRNA, reads ending at DCL1 cleavage site (co-transcriptionally processed) and those expanding the site (unprocessed pri-miRNAs) were used (Figure 4A). For each independent experiment analyzed, the output files in bigWig format (plaNET-seq for co-transcriptional processing, and full coverage of re-mapped plaNET-seq reads for unprocessed pri-miRNAs) were used to calculate the scores for specific genomic regions (DCL1 cuts) using deepTools multiBigwigSummary in BED-file mode (Ramirez et al., 2016). In order to simplify the analysis, MIRNA loci with low plaNET-seq signal and with unclear processing mechanisms were excluded. The outputs of multiBigWigSummary were 
processed to obtain the ratios of pri-miRNAs in each condition and sorted by processing type in the $\mathrm{R}$ statistical programming environment ( $\mathrm{R}$ Core Team, 2020), and graphics were produced with the ggplot package. Snapshots of the mapped plaNET-seq reads were constructed using the Integrative Genomics Viewer(Robinson et al., 2011).

\section{Probes preparation}

For the detection of pri-miRNAs, we applied antisense DNA oligonucleotides labeled with digoxigenin at their 5' ends and hybridizing to different segments of pri-miRNA163 and pri-miRNA156a. We designed probes targeting: an intron located downstream of the stem-loop structure (Intron), an exon (Exon), and two joined exons (Exon/Exon) as well as a loop sequence (Loop), a miRNA star (miRNA*) and a mature miRNA (miRNA) (check Table S1 for probe sequences). Next, we used Terminal Transferase Reaction to add an additional nucleotide conjugated with digoxigenin to the 3 ' end of each probe according to the protocol delivered by Thermo-Fisher. The probes were incubated in reaction buffer $\left(5 \mathrm{mM} \mathrm{CoCl}_{2}, 400 \mathrm{U} /\right.$ reaction of Terminal Transferase (Sigma Merck) 0.1 mM DIG-11-dUTP (Sigma Merck), 0.1 mM dATP, 0.2 mM Alexa Fluor 488-5-dUTP (Thermo Fisher), probe final concentration: $10 \mathrm{pM}$, for $40 \mathrm{~min}$. at $37^{\circ}$ C.

RNA Stellaris probes were designed by using software: Stellaris Probe Designer version 2.0 from Biosearch Technologies. The probes used were selected to target the intron sequence located downstream of the stem-loop structure of primiRNA156a and labeled with Quasar 570 or fluoresceine (6-FAM).

\section{Combined FISH and immunolocalization}

pri-miRNAs were localized by applying fluorescence in situ hybridization (FISH) combined with the immunolocalization of digoxigenin attached to 5' and 3' ends of the probes. In our experiments FISH preceded the immunocytochemical methods. Prior to the assay, the cells were treated with PBS buffer containing 0.1 \% Triton X-100 for cell membrane permeabilization. Next, the cells were hybridized with the probes in hybridization buffer $(30 \%(\mathrm{v} / \mathrm{v})$ formamide, $4 \times$ SSC (600 mM NaCl, $6 \mu \mathrm{M}$ sodium citrate), $5 \times$ Denhardt's solution $(0.1 \%(\mathrm{~g} / \mathrm{v})$ ficoll 
400, $0.1 \%(\mathrm{~g} / \mathrm{v})$ polyvinilpyrolidone, $0.1 \%(\mathrm{~g} / \mathrm{v}) \mathrm{BSA}), 1 \mathrm{mM}$ EDTA, and 50mM phosphate buffer) in a humified chamber for overnight at $26{ }^{\circ} \mathrm{C}$. After washing, we applied the immunolabeling assay using primary mouse (Sigma Merck) or rabbit (Sigma Merck) anti-DIG antibodies (diluted 1:100) in 0.05\% acetylated BSA in PBS for overnight at $10^{\circ} \mathrm{C}$. Subsequently, the cells were washed with PBS and subjected to incubation with goat anti-mouse or goat anti-rabbit secondary antibodies conjugated with Alexa Fluor 488 or Alexa Fluor 555 (Thermo Fisher) in $0.05 \%$ acetylated BSA in PBS for $2 \mathrm{~h}$ at $37^{\circ} \mathrm{C}$. DNA was stained with Hoechst 33342 (Thermo Fisher) and mounted in ProLong Gold antifade reagent (Life Technologies).

\section{Immunodetection of proteins}

Double immunodetection experiments were performed according to Bhat et al. (2020). The isolated nuclei were treated with PBS containing $0.1 \%$ Triton $\mathrm{X}-100$ and next incubated with primary antibodies in $0.05 \%$ acetylated BSA in PBS for overnight at $10{ }^{\circ} \mathrm{C}$. In our experiment we used antibodies targeting: SERRATE (Agrisera, diluted 1:100), HYL1 (Agrisera, diluted 1:200) and DCL-1 (Agrisera, diluted 1:100). For the localization of RNAPII we used antibodies recognizing RNAPII phosphorylated at serine 5 (Chromotek, diluted 1:200) and serine 2 (Chromotek, diluted 1:200). After washing with PBS, the slides were incubated with secondary goat anti-rabbit or goat anti-rat antibodies conjugated with Alexa Fluor 488 or Alexa 555 (Thermo Fisher, diluted 1:100) in PBS containing $0.01 \%$ acetylated BSA at $37^{\circ} \mathrm{C}$ for $2 \mathrm{~h}$. Next, the slides were stained for DNA detection with Hoechst 33342 (Thermo Fisher), and mounted in ProLong Gold antifade reagent (Life Technologies).

\section{Microscopic and correlation analysis}

The results were registered with the Leica SP8 confocal microscope using lasers emitting light at wavelengths of 405, 488 and $561 \mathrm{~nm}$ with an optimized pinhole, long exposure time $(200 \mathrm{kHz})$ and 63x (numerical aperture, 1.4). For the Leica confocal microscope Plan Apochromat DIC H an oil immersion lens was used. To minimize bleed-through between fluorescence channels, the low laser power $(0.4-5 \%$ of maximum power) and single-channel collection were applied. 
Correlation analyses were performed with the use of Pearson's correlation coefficient, Spearman's rank correlation and ICQ value by using free software ImageJ from the National Institute of Health in USA, and its plugin coloc2 which is the analysis option of the expanded ImageJ version Fiji.

\section{Author contribution}

L.G, I.T, T.G, Z.S-K, A.J and P.A.M conceived and designed the study. L.G, performed all IP experiments and validations; I.T. analyzed all the sequencing data; T.G. and A.K-M performed all microscopic experiments; D.A.C performed selected HST experiments; J.D.S designed and supervised microscopic experiments; P.A.M, A.J, Z.Sz-K and S.M supervised the work and secured project funding; L.G, I.T, T.G, Z.Sz-K, A.J and P.A.M wrote the manuscript with input from all authors.

\section{Acknowledgments}

This work was supported by grants from ANPCyT (Agencia Nacional de Promoción Científica y Tecnológica, Argentina) and the Polish National Science Centre (UMO-2019/32/T/NZ1/00508, UMO-2016/23/N/NZ1/00010, UMO2013/10/A/NZ1/00557). The authors also received financial support from the Initiative of Excellence-Research University (05/IDUB/2019/94) at Adam Mickiewicz University, Poznan, Poland. P.A.M. and D.A.C. are members of CONICET; L.G is a fellow of the same institution. We would like to thanks Federico D. Ariel for his valuable comments on this project and Dr. Pauline Julline (Institute of Plant Science, University of Bern, Switzerland) for sharing with us seeds of transgenic plants expressing mCherry-AGO1

\section{Footnotes}

The authors declare no competing interest.

\section{References}

Achkar, N.P., Cambiagno, D.A., and Manavella, P.A. (2016). miRNA Biogenesis: A Dynamic Pathway. Trends Plant Sci 21, 1034-1044. 10.1016/j.tplants.2016.09.003. 
Addo-Quaye, C., Snyder, J.A., Park, Y.B., Li, Y.F., Sunkar, R., and Axtell, M.J. (2009). Sliced microRNA targets and precise loop-first processing of MIR319 hairpins revealed by analysis of the Physcomitrella patens degradome. RNA 15, 2112-2121. 10.1261/rna.1774909.

Aguilera, A., and Garcia-Muse, T. (2012). R loops: from transcription byproducts to threats to genome stability. Mol Cell 46, 115-124. 10.1016/j.molcel.2012.04.009.

Akhtar, J., Renaud, Y., Albrecht, S., Ghavi-Helm, Y., Roignant, J.Y., Silies, M., and Junion, G. (2021). m(6)A RNA methylation regulates promoter- proximal pausing of RNA polymerase II. Mol Cell. 10.1016/j.molcel.2021.06.023.

Ariel, F., Lucero, L., Christ, A., Mammarella, M.F., Jegu, T., Veluchamy, A., Mariappan, K., Latrasse, D., Blein, T., Liu, C., et al. (2020). R-Loop Mediated trans Action of the APOLO Long Noncoding RNA. Mol Cell 77, 1055-1065 e1054. 10.1016/j.molcel.2019.12.015.

Axtell, M.J., Westholm, J.O., and Lai, E.C. (2011). Vive la difference: biogenesis and evolution of microRNAs in plants and animals. Genome Biol 12, 221. 10.1186/gb-2011-12-4-221.

Ballarino, M., Pagano, F., Girardi, E., Morlando, M., Cacchiarelli, D., Marchioni, M., Proudfoot, N.J., and Bozzoni, I. (2009). Coupled RNA processing and transcription of intergenic primary microRNAs. Mol Cell Biol 29, 5632-5638. 10.1128/MCB.00664-09.

Baranauske, S., Mickute, M., Plotnikova, A., Finke, A., Venclovas, C., Klimasauskas, S., and Vilkaitis, G. (2015). Functional mapping of the plant small RNA methyltransferase: HEN1 physically interacts with HYL1 and DICER-LIKE 1 proteins. Nucleic Acids Res 43, 2802-2812. 10.1093/nar/gkv102.

Bartel, D.P. (2018). Metazoan MicroRNAs. Cell 173, 20-51. 10.1016/j.cell.2018.03.006.

Bentley, D.L. (2014). Coupling mRNA processing with transcription in time and space. Nat Rev Genet 15, 163-175. 10.1038/nrg3662.

Bhat, S.S., Bielewicz, D., Gulanicz, T., Bodi, Z., Yu, X., Anderson, S.J., Szewc, L., Bajczyk, M., Dolata, J., Grzelak, N., et al. (2020). mRNA adenosine methylase (MTA) deposits $\mathrm{m}(6) \mathrm{A}$ on pri-miRNAs to modulate miRNA biogenesis in Arabidopsis thaliana. Proc Natl Acad Sci U S A 117, 21785-21795. 10.1073/pnas.2003733117. 
Bologna, N.G., Mateos, J.L., Bresso, E.G., and Palatnik, J.F. (2009). A loop-to-base processing mechanism underlies the biogenesis of plant microRNAs miR319 and miR159. EMBO J 28, 3646-3656. 10.1038/emboj.2009.292.

Bologna, N.G., Schapire, A.L., Zhai, J., Chorostecki, U., Boisbouvier, J., Meyers, B.C., and Palatnik, J.F. (2013). Multiple RNA recognition patterns during microRNA biogenesis in plants. Genome Res 23, 1675-1689. 10.1101/gr.153387.112.

Brioudes, F., Jay, F., Sarazin, A., Grentzinger, T., Devers, E.A., and Voinnet, O. (2021). HASTY, the Arabidopsis EXPORTIN5 ortholog, regulates cell-to-cell and vascular microRNA movement. EMBO J 40, e107455. 10.15252/embj.2020107455.

Brosnan, C.A., Sarazin, A., Lim, P., Bologna, N.G., Hirsch-Hoffmann, M., and Voinnet, O. (2019). Genome-scale, single-cell-type resolution of microRNA activities within a whole plant organ. EMBO J 38, e100754. 10.15252/embj.2018100754.

Cambiagno, D.A., Giudicatti, A.J., Arce, A.L., Gagliardi, D., Li, L., Yuan, W., Lundberg, D.S., Weigel, D., and Manavella, P.A. (2021). HASTY modulates miRNA biogenesis by linking pri-miRNA transcription and processing. Mol Plant 14, 426-439. 10.1016/j.molp.2020.12.019.

Campbell, M.S., Law, M., Holt, C., Stein, J.C., Moghe, G.D., Hufnagel, D.E., Lei, J., Achawanantakun, R., Jiao, D., Lawrence, C.J., et al. (2014). MAKER-P: a tool kit for the rapid creation, management, and quality control of plant genome annotations. Plant Physiol 164, 513-524. 10.1104/pp.113.230144.

Chedin, F. (2016). Nascent Connections: R-Loops and Chromatin Patterning. Trends Genet 32, 828-838. 10.1016/j.tig.2016.10.002.

Crossley, M.P., Bocek, M., and Cimprich, K.A. (2019). R-Loops as Cellular Regulators and Genomic Threats. Mol Cell 73, 398-411. 10.1016/j.molcel.2019.01.024.

Cui, Y., Fang, X., and Qi, Y. (2016). TRANSPORTIN1 Promotes the Association of MicroRNA with ARGONAUTE1 in Arabidopsis. Plant Cell 28, 2576-2585. 10.1105/tpc.16.00384. 
Cuperus, J.T., Fahlgren, N., and Carrington, J.C. (2011). Evolution and functional diversification of MIRNA genes. Plant Cell 23, 431-442. 10.1105/tpc.110.082784.

de la Mata, M., Lafaille, C., and Kornblihtt, A.R. (2010). First come, first served revisited: factors affecting the same alternative splicing event have different effects on the relative rates of intron removal. RNA 16, 904-912. 10.1261/rna.1993510.

Devers, E.A., Brosnan, C.A., Sarazin, A., Albertini, D., Amsler, A.C., Brioudes, F., Jullien, P.E., Lim, P., Schott, G., and Voinnet, O. (2020). Movement and differential consumption of short interfering RNA duplexes underlie mobile RNA interference. Nat Plants 6, 789-799. 10.1038/s41477-020-0687-2.

Dong, Z., Han, M.H., and Fedoroff, N. (2008). The RNA-binding proteins HYL1 and SE promote accurate in vitro processing of pri-miRNA by DCL1. Proc Natl Acad Sci U S A 105, 9970-9975. 10.1073/pnas.0803356105.

Dujardin, G., Lafaille, C., de la Mata, M., Marasco, L.E., Munoz, M.J., Le Jossic-Corcos, C., Corcos, L., and Kornblintt, A.R. (2014). How slow RNA polymerase II elongation favors alternative exon skipping. Mol Cell 54, 683-690. 10.1016/j.molcel.2014.03.044.

Fan, L., Zhang, C., Zhang, Y., Stewart, E., Jez, J., Nakajima, K., and Chen, X. (2021). Microtubules Promote the Non-cell Autonomy of MicroRNAs by Inhibiting their Cytoplasmic Loading into ARGONAUTE1 in <em>Arabidopsis</em>. bioRxiv, 2021.2005.2026.445899. 10.1101/2021.05.26.445899.

Fang, X., Cui, Y., Li, Y., and Qi, Y. (2015). Transcription and processing of primary microRNAs are coupled by Elongator complex in Arabidopsis. Nat Plants 1, 15075. 10.1038/nplants.2015.75.

Ginno, P.A., Lott, P.L., Christensen, H.C., Korf, I., and Chedin, F. (2012). $\mathrm{R}$-loop formation is a distinctive characteristic of unmethylated human $\mathrm{CpG}$ island promoters. Mol Cell 45, 814-825. 10.1016/j.molcel.2012.01.017.

Giono, L.E., and Kornblihtt, A.R. (2020). Linking transcription, RNA polymerase II elongation and alternative splicing. Biochem J 477, 3091-3104. 10.1042/BCJ20200475. 
Godoy Herz, M.A., and Kornblihtt, A.R. (2019). Alternative Splicing and Transcription Elongation in Plants. Front Plant Sci 10, 309. 10.3389/fpls.2019.00309.

Hamperl, S., and Cimprich, K.A. (2014). The contribution of cotranscriptional RNA:DNA hybrid structures to DNA damage and genome instability. DNA Repair (Amst) 19, 84-94. 10.1016/j.dnarep.2014.03.023.

Herzel, L., Ottoz, D.S.M., Alpert, T., and Neugebauer, K.M. (2017). Splicing and transcription touch base: co-transcriptional spliceosome assembly and function. Nat Rev Mol Cell Biol 18, 637-650. 10.1038/nrm.2017.63.

Huang, V., Zheng, J., Qi, Z., Wang, J., Place, R.F., Yu, J., Li, H., and Li, L.C. (2013). Ago1 Interacts with RNA polymerase II and binds to the promoters of actively transcribed genes in human cancer cells. PLoS Genet 9, e1003821. 10.1371/journal.pgen.1003821.

Kim, Y.J., Zheng, B., Yu, Y., Won, S.Y., Mo, B., and Chen, X. (2011). The role of Mediator in small and long noncoding RNA production in Arabidopsis thaliana. EMBO J 30, 814-822. 10.1038/emboj.2011.3.

Kindgren, P., Ivanov, M., and Marquardt, S. (2020). Native elongation transcript sequencing reveals temperature dependent dynamics of nascent RNAPII transcription in Arabidopsis. Nucleic Acids Res 48, 2332-2347. 10.1093/nar/gkz1189.

Knop, K., Stepien, A., Barciszewska-Pacak, M., Taube, M., Bielewicz, D., Michalak, M., Borst, J.W., Jarmolowski, A., and Szweykowska-Kulinska, Z. (2017). Active 5' splice sites regulate the biogenesis efficiency of Arabidopsis microRNAs derived from intron-containing genes. Nucleic Acids Res 45, 27572775. 10.1093/nar/gkw895.

Knuckles, P., Carl, S.H., Musheev, M., Niehrs, C., Wenger, A., and Buhler, M. (2017). RNA fate determination through cotranscriptional adenosine methylation and microprocessor binding. Nat Struct Mol Biol 24, 561-569. 10.1038/nsmb.3419.

Kozomara, A., Birgaoanu, M., and Griffiths-Jones, S. (2019). miRBase: from microRNA sequences to function. Nucleic Acids Res 47, D155-D162. 10.1093/nar/gky1141. 
Lauressergues, D., Couzigou, J.M., Clemente, H.S., Martinez, Y., Dunand, C., Becard, G., and Combier, J.P. (2015). Primary transcripts of microRNAs encode regulatory peptides. Nature 520, 90-93. 10.1038/nature14346.

Lee, K.M., and Tarn, W.Y. (2013). Coupling pre-mRNA processing to transcription on the RNA factory assembly line. RNA Biol 10, 380-390. 10.4161/rna.23697.

Leng, X., Ivanov, M., Kindgren, P., Malik, I., Thieffry, A., Brodersen, P., Sandelin, A., Kaplan, C.D., and Marquardt, S. (2020). Organismal benefits of transcription speed control at gene boundaries. EMBO Rep 21, e49315. 10.15252/embr.201949315.

Liu, C., Xin, Y., Xu, L., Cai, Z., Xue, Y., Liu, Y., Xie, D., Liu, Y., and Qi, Y. (2018). Arabidopsis ARGONAUTE 1 Binds Chromatin to Promote Gene Transcription in Response to Hormones and Stresses. Dev Cell 44, 348-361 e347. 10.1016/j.devcel.2017.12.002.

Llave, C., Xie, Z., Kasschau, K.D., and Carrington, J.C. (2002). Cleavage of Scarecrow-like mRNA targets directed by a class of Arabidopsis miRNA. Science 297, 2053-2056. 10.1126/science.1076311.

Lunardon, A., Kariuki, S.M., and Axtell, M.J. (2021). Expression and processing of polycistronic artificial microRNAs and trans-acting siRNAs from transiently introduced transgenes in Solanum lycopersicum and Nicotiana benthamiana. Plant J 106, 1087-1104. 10.1111/tpj.15221.

Mi, S., Cai, T., Hu, Y., Chen, Y., Hodges, E., Ni, F., Wu, L., Li, S., Zhou, H., Long, C., et al. (2008). Sorting of small RNAs into Arabidopsis argonaute complexes is directed by the $5^{\prime}$ terminal nucleotide. Cell $133,116-127$. 10.1016/j.cell.2008.02.034.

Miguel, V.N., Manavella, P.A., Chan, R.L., and Capella, M.A. (2020). The AtHB1 Transcription Factor Controls the miR164-CUC2 Regulatory Node to Modulate Leaf Development. Plant Cell Physiol 61, 659-670. 10.1093/pcp/pcz233.

Morlando, M., Ballarino, M., Gromak, N., Pagano, F., Bozzoni, I., and Proudfoot, N.J. (2008). Primary microRNA transcripts are processed cotranscriptionally. Nat Struct Mol Biol 15, 902-909. 10.1038/nsmb.1475.

Moro, B., Chorostecki, U., Arikit, S., Suarez, I.P., Hobartner, C., Rasia, R.M., Meyers, B.C., and Palatnik, J.F. (2018). Efficiency and precision of 
microRNA biogenesis modes in plants. Nucleic Acids Res 46, 10709-10723. 10.1093/nar/gky853.

Morton, T., Petricka, J., Corcoran, D.L., Li, S., Winter, C.M., Carda, A., Benfey, P.N., Ohler, U., and Megraw, M. (2014). Paired-end analysis of transcription start sites in Arabidopsis reveals plant-specific promoter signatures. Plant Cell 26, 2746-2760. 10.1105/tpc.114.125617.

Muniz, L., Nicolas, E., and Trouche, D. (2021). RNA polymerase II speed: a key player in controlling and adapting transcriptome composition. EMBO J 40, e105740. 10.15252/embj.2020105740.

Niedojadlo, K., Piecinski, S., Smolinski, D.J., and Bednarska-Kozakiewicz, E. (2012). Transcriptional activity of Hyacinthus orientalis L. female gametophyte cells before and after fertilization. Planta 236, 153-169. 10.1007/s00425-0121599-9.

Niehrs, C., and Luke, B. (2020). Regulatory R-loops as facilitators of gene expression and genome stability. Nat Rev Mol Cell Biol 21, 167-178. 10.1038/s41580-019-0206-3.

Nielsen, M., Ard, R., Leng, X., Ivanov, M., Kindgren, P., Pelechano, V., and Marquardt, S. (2019). Transcription-driven chromatin repression of Intragenic transcription start sites. PLoS Genet 15, e1007969. 10.1371/journal.pgen.1007969.

Nojima, T., Gomes, T., Grosso, A.R.F., Kimura, H., Dye, M.J., Dhir, S., Carmo-Fonseca, M., and Proudfoot, N.J. (2015). Mammalian NET-Seq Reveals Genome-wide Nascent Transcription Coupled to RNA Processing. Cell 161, 526540. 10.1016/j.cell.2015.03.027.

Onodera, Y., Nakagawa, K., Haag, J.R., Pikaard, D., Mikami, T., Ream, T., Ito, Y., and Pikaard, C.S. (2008). Sex-biased lethality or transmission of defective transcription machinery in Arabidopsis. Genetics 180, 207-218. 10.1534/genetics.108.090621.

Pawlicki, J.M., and Steitz, J.A. (2008). Primary microRNA transcript retention at sites of transcription leads to enhanced microRNA production. J Cell Biol 182, 61-76. 10.1083/jcb.200803111.

Peck, S.A., Hughes, K.D., Victorino, J.F., and Mosley, A.L. (2019). Writing a wrong: Coupled RNA polymerase II transcription and RNA quality control. Wiley Interdiscip Rev RNA 10, e1529. 10.1002/wrna.1529. 
Ramirez, F., Ryan, D.P., Gruning, B., Bhardwaj, V., Kilpert, F., Richter, A.S., Heyne, S., Dundar, F., and Manke, T. (2016). deepTools2: a next generation web server for deep-sequencing data analysis. Nucleic Acids Res 44, W160-165. 10.1093/nar/gkw257.

Re, D.A., Lang, P.L.M., Yones, C., Arce, A.L., Stegmayer, G., Milone, D., and Manavella, P.A. (2019). Alternative use of miRNA-biogenesis co-factors in plants at low temperatures. Development 146. 10.1242/dev.172932.

Robinson, J.T., Thorvaldsdottir, H., Winckler, W., Guttman, M., Lander, E.S., Getz, G., and Mesirov, J.P. (2011). Integrative genomics viewer. Nat Biotechnol 29, 24-26. 10.1038/nbt.1754.

Salataj, E., Stathopoulou, C., Hafthornorsson, R.A., Nikolaou, C., and Spilianakis, C.G. (2019). Developmental conservation of microRNA gene localization at the nuclear periphery. PLoS One 14, e0223759. 10.1371/journal.pone.0223759.

Saldi, T., Fong, N., and Bentley, D.L. (2018). Transcription elongation rate affects nascent histone pre-mRNA folding and 3' end processing. Genes Dev 32, 297-308. 10.1101/gad.310896.117.

Santos-Pereira, J.M., and Aguilera, A. (2015). R loops: new modulators of genome dynamics and function. Nat Rev Genet 16, 583-597. 10.1038/nrg3961.

Sanz, L.A., Hartono, S.R., Lim, Y.W., Steyaert, S., Rajpurkar, A., Ginno, P.A., Xu, X., and Chedin, F. (2016). Prevalent, Dynamic, and Conserved R-Loop Structures Associate with Specific Epigenomic Signatures in Mammals. Mol Cell 63, 167-178. 10.1016/j.molcel.2016.05.032.

Scharfen, L., and Neugebauer, K.M. (2021). Transcription Regulation Through Nascent RNA Folding. J Mol Biol 433, 166975. 10.1016/j.jmb.2021.166975.

Shafiq, S., Chen, C., Yang, J., Cheng, L., Ma, F., Widemann, E., and Sun, Q. (2017). DNA Topoisomerase 1 Prevents R-loop Accumulation to Modulate Auxin-Regulated Root Development in Rice. Mol Plant 10, 821-833. 10.1016/j.molp.2017.04.001.

Singh, A.K., Singh, N., Kumar, S., Kumari, J., Singh, R., Gaba, S., Yadav, M.C., Grover, M., Chaurasia, S., and Kumar, R. (2020). Identification and evolutionary analysis of polycistronic miRNA clusters in domesticated and wild wheat. Genomics 112, 2334-2348. 10.1016/j.ygeno.2020.01.005. 
Song, L., Han, M.H., Lesicka, J., and Fedoroff, N. (2007). Arabidopsis primary microRNA processing proteins HYL1 and DCL1 define a nuclear body distinct from the Cajal body. Proc Natl Acad Sci U S A 104, 5437-5442. 10.1073/pnas.0701061104.

Speth, C., Szabo, E.X., Martinho, C., Collani, S., Zur Oven-Krockhaus, S., Richter, S., Droste-Borel, I., Macek, B., Stierhof, Y.D., Schmid, M., et al. (2018). Arabidopsis RNA processing factor SERRATE regulates the transcription of intronless genes. Elife 7. 10.7554/eLife.37078.

Sun, Q., Csorba, T., Skourti-Stathaki, K., Proudfoot, N.J., and Dean, C. (2013). R-loop stabilization represses antisense transcription at the Arabidopsis FLC locus. Science 340, 619-621. 10.1126/science.1234848.

Szarzynska, B., Sobkowiak, L., Pant, B.D., Balazadeh, S., Scheible, W.R., Mueller-Roeber, B., Jarmolowski, A., and Szweykowska-Kulinska, Z. (2009). Gene structures and processing of Arabidopsis thaliana HYL1-dependent primiRNAs. Nucleic Acids Res 37, 3083-3093. 10.1093/nar/gkp189.

Tomassi, A.H., Re, D.A., Romani, F., Cambiagno, D.A., Gonzalo, L., Moreno, J.E., Arce, A.L., and Manavella, P.A. (2020). The Intrinsically Disordered Protein CARP9 Bridges HYL1 to AGO1 in the Nucleus to Promote MicroRNA Activity. Plant Physiol 184, 316-329. 10.1104/pp.20.00258.

Turunen, T.A., Roberts, T.C., Laitinen, P., Vaananen, M.A., Korhonen, P., Malm, T., Yla-Herttuala, S., and Turunen, M.P. (2019). Changes in nuclear and cytoplasmic microRNA distribution in response to hypoxic stress. Sci Rep 9, 10332. 10.1038/s41598-019-46841-1.

Wang, L., Song, X., Gu, L., Li, X., Cao, S., Chu, C., Cui, X., Chen, X., and Cao, X. (2013). NOT2 proteins promote polymerase II-dependent transcription and interact with multiple MicroRNA biogenesis factors in Arabidopsis. Plant Cell 25, 715-727. 10.1105/tpc.112.105882.

Wang, Z., Ma, Z., Castillo-Gonzalez, C., Sun, D., Li, Y., Yu, B., Zhao, B., Li, P., and Zhang, X. (2018). SWI2/SNF2 ATPase CHR2 remodels pri-miRNAs via Serrate to impede miRNA production. Nature 557, 516-521. 10.1038/s41586018-0135-x.

Xie, D., Chen, M., Niu, J., Wang, L., Li, Y., Fang, X., Li, P., and Qi, Y. (2021). Phase separation of SERRATE drives dicing body assembly and 
promotes miRNA processing in Arabidopsis. Nat Cell Biol 23, 32-39. 10.1038/s41556-020-00606-5.

Xie, Z., Allen, E., Fahlgren, N., Calamar, A., Givan, S.A., and Carrington, J.C. (2005). Expression of Arabidopsis MIRNA genes. Plant Physiol 138, 21452154. 10.1104/pp.105.062943.

Xu, W., Li, K., Li, S., Hou, Q., Zhang, Y., Liu, K., and Sun, Q. (2020). The R-Loop Atlas of Arabidopsis Development and Responses to Environmental Stimuli. Plant Cell 32, 888-903. 10.1105/tpc.19.00802.

Xu, W., Xu, H., Li, K., Fan, Y., Liu, Y., Yang, X., and Sun, Q. (2017). The R-loop is a common chromatin feature of the Arabidopsis genome. Nat Plants 3 , 704-714. 10.1038/s41477-017-0004-x.

Yang, J., Cao, Y., and Ma, L. (2021). Co-Transcriptional RNA Processing in Plants: Exploring from the Perspective of Polyadenylation. Int J Mol Sci 22. 10.3390/ijms22073300.

Yang, S.W., Chen, H.Y., Yang, J., Machida, S., Chua, N.H., and Yuan, Y.A. (2010). Structure of Arabidopsis HYPONASTIC LEAVES1 and its molecular implications for miRNA processing. Structure 18, 594-605. 10.1016/j.str.2010.02.006.

Yang, X., Ren, W., Zhao, Q., Zhang, P., Wu, F., and He, Y. (2014). Homodimerization of HYL1 ensures the correct selection of cleavage sites in primary miRNA. Nucleic Acids Res 42, 12224-12236. 10.1093/nar/gku907.

Yang, Z., Hou, Q., Cheng, L., Xu, W., Hong, Y., Li, S., and Sun, Q. (2017). RNase H1 Cooperates with DNA Gyrases to Restrict R-Loops and Maintain Genome Integrity in Arabidopsis Chloroplasts. Plant Cell 29, 2478-2497. 10.1105/tpc.17.00305.

Yang, Z., Li, M., and Sun, Q. (2020). RHON1 Co-transcriptionally Resolves R-Loops for Arabidopsis Chloroplast Genome Maintenance. Cell Rep 30, 243256 e245. 10.1016/j.celrep.2019.12.007.

You, Q., Yan, H., Liu, Y., Yi, X., Zhang, K., Xu, W., and Su, Z. (2017). A systemic identification approach for primary transcription start site of Arabidopsis miRNAs from multidimensional omics data. Funct Integr Genomics 17, 353-363. 10.1007/s10142-016-0541-9. 
Yuan, W., Zhou, J., Tong, J., Zhuo, W., Wang, L., Li, Y., Sun, Q., and Qian, $W$. (2019). ALBA protein complex reads genic R-loops to maintain genome stability in Arabidopsis. Sci Adv 5, eaav9040. 10.1126/sciadv.aav9040.

Zatreanu, D., Han, Z., Mitter, R., Tumini, E., Williams, H., Gregersen, L., Dirac-Svejstrup, A.B., Roma, S., Stewart, A., Aguilera, A., and Svejstrup, J.Q. (2019). Elongation Factor TFIIS Prevents Transcription Stress and R-Loop Accumulation to Maintain Genome Stability. Mol Cell 76, 57-69 e59. 10.1016/j.molcel.2019.07.037.

Zhang, W., Gao, S., Zhou, X., Xia, J., Chellappan, P., Zhou, X., Zhang, X., and Jin, H. (2010). Multiple distinct small RNAs originate from the same microRNA precursors. Genome Biol 11, R81. 10.1186/gb-2010-11-8-r81.

Zhang, Y., Yun, Z., Gong, L., Qu, H., Duan, X., Jiang, Y., and Zhu, H. (2018). Comparison of miRNA Evolution and Function in Plants and Animals. Microrna 7, 4-10. 10.2174/2211536607666180126163031.

Zhu, H., Zhou, Y., Castillo-Gonzalez, C., Lu, A., Ge, C., Zhao, Y.T., Duan, L., Li, Z., Axtell, M.J., Wang, X.J., and Zhang, X. (2013). Bidirectional processing of pri-miRNAs with branched terminal loops by Arabidopsis Dicer-like1. Nat Struct Mol Biol 20, 1106-1115. 10.1038/nsmb.2646. 


\section{Figure legends}

A
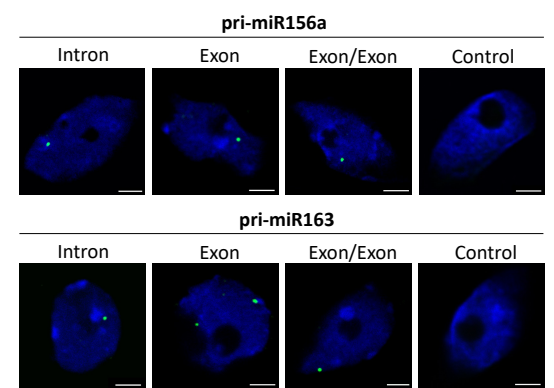

C
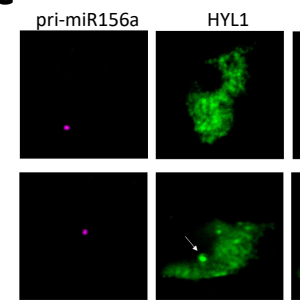

pri-miR156a

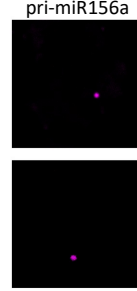

D
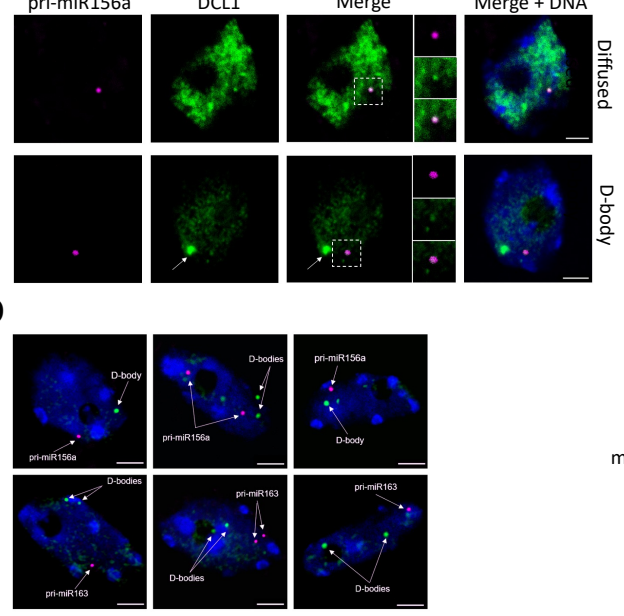

$\overbrace{0-\text {-bodes }}$

B

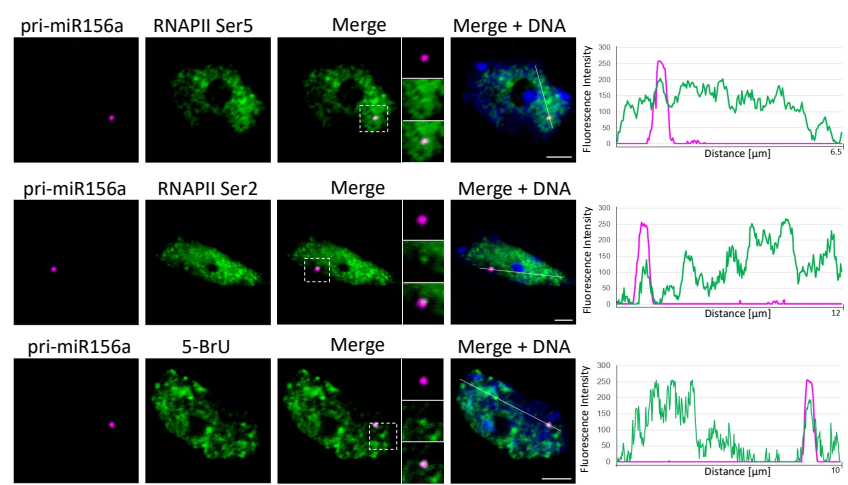

E pri-miR156a
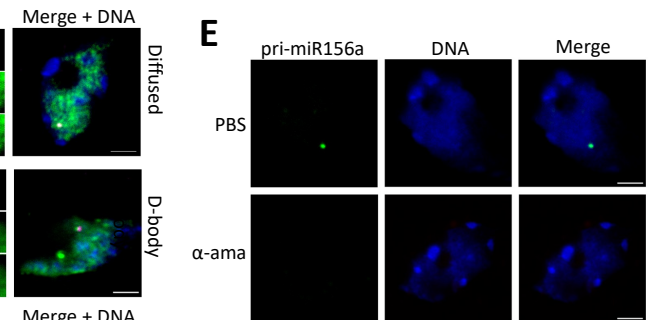

$\mathbf{F}$

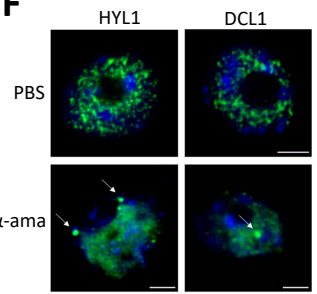

G

Merge + DNA
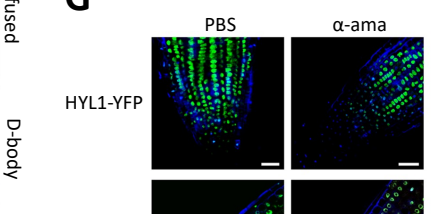

HYL1-YFP

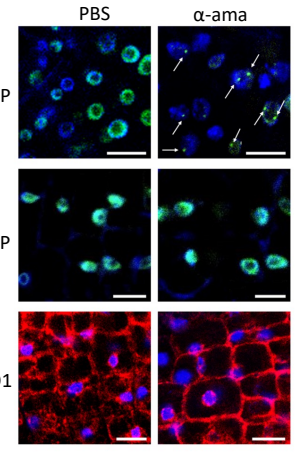

mCherry-AGO1
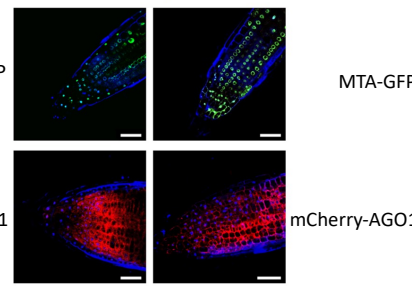

Figure 1. Subcellular distribution and interactions of nascent primiRNAs and the miRNA biogenesis complex.

(A) FISH of pri-miRNA156a and pri-miR163 (green) using mouse antibodies targeting digoxigenin in the nuclei isolated from wild-type plant cells. The probes hybridizing to intron (Intron), exon (Exon), transcripts after splicing (Exon/Exon); hybridization buffer without any probe was used as a negative control (Control).

(B) Detection of pri-miR156a using RNA Stellaris probes (magenta) and RNAPII Ser5, RNAPII Ser2, or newly synthesized transcripts (green). On the right are shown the fluorescence intensity plotted along the white line shown each panel on the left. The fluorescence intensity of pri-miR156a is depicted as the 
magenta curves, while RNAPII Ser 5, RNAPII Ser 2, and 5-BrU as the green curves.

(C and D) FISH of pri-miRNA156a (magenta) combined with immunolabeling of HYL1 (C) or DCL1 (C and D).

(E and F) FISH of pri-miRNA156a using RNA Stellaris (green) (E) and immunodetection of HYL1 or DCL-1 (green) $(F)$ in plants treated with a-amanitin or PBS as a negative control. White arrows mark D-Bodies.

In all cases, the nuclei were stained with Hoechst (blue). Scale bar - $2.5 \mu \mathrm{m}$.

(G) HYL1-YFP distribution in the root cells of the meristematic zone in plants treated with buffer (PBS) (upper panel) and a-amanitin (bottom panel). In the right is the magnification of representative images obtained from the root meristematic zone cells of four different plants. White arrows mark D-Bodies. Scale bar: $10 \mu \mathrm{m}$. DNA was stained with Hoechst (blue).
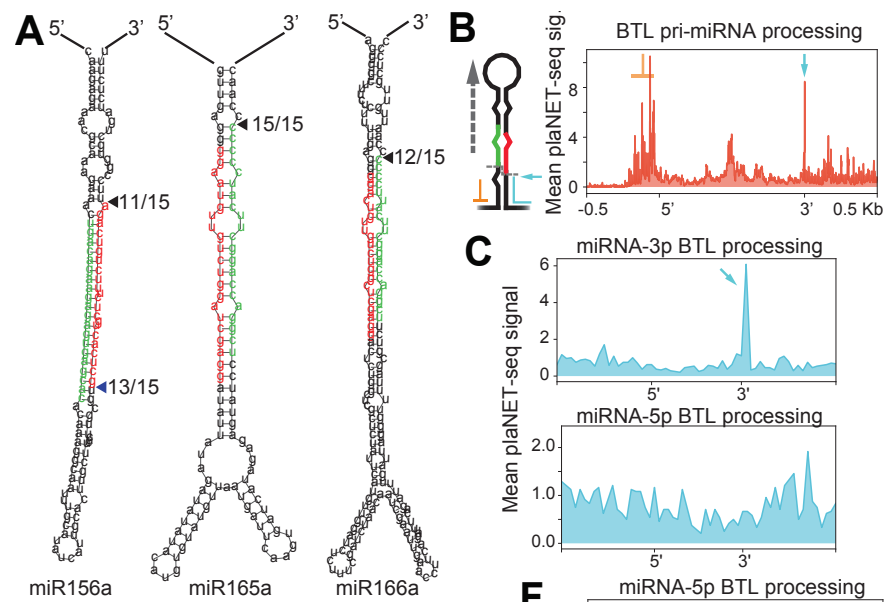

C
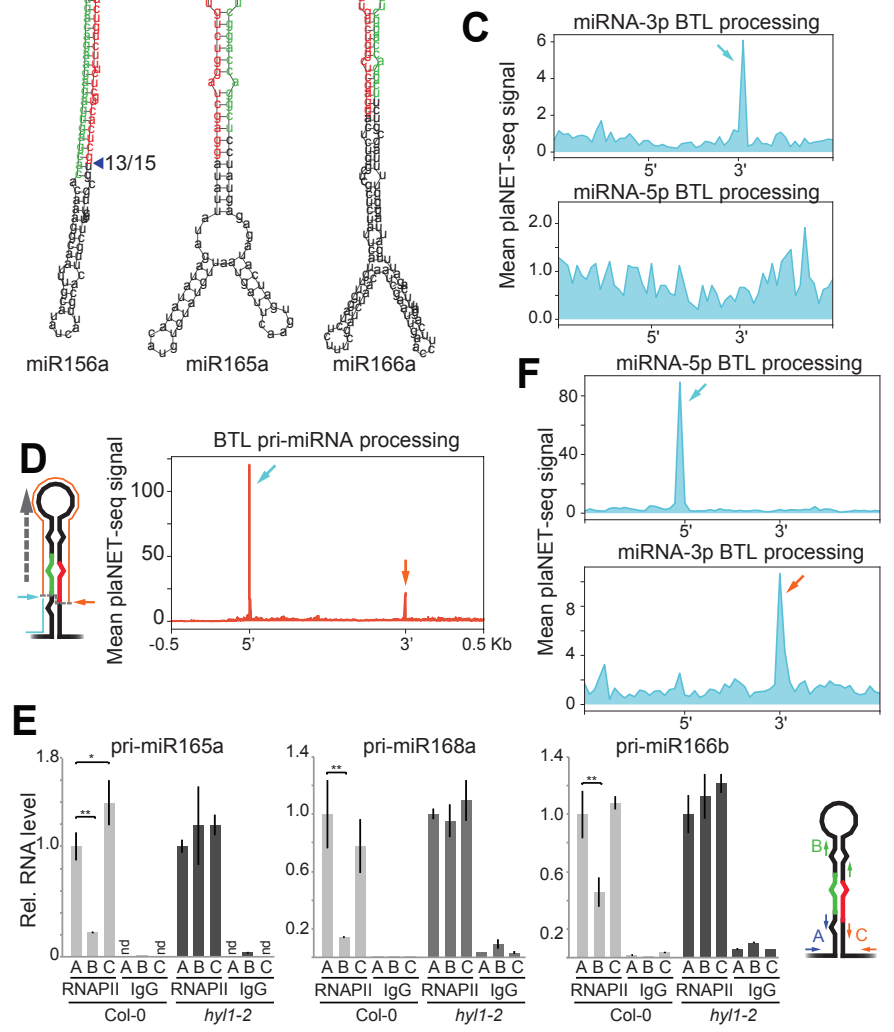

Figure 2. Plant pri-miRNAs are processed co-transcriptionally 
(A) Detection of nascent pri-miRNA processing intermediates associated with RNAPII as detected by 5'RACE. The fraction of clones with the expected 5' end (among all the sequenced products) is indicated next to the predicted primiRNAs secondary structures. The miRNA-5p and miRNA-3p positions are noted in red and green respectively.

( $B, C, D$, and $F$ ) Metagene analysis of nascent $B T L$ pri-miRNAs processing intermediated associated to RNAPII as determined by scoring the 3'-end nucleotide in of plaNET-seq reads in the plus and minus strands of plaNETseq samples ( $B$ and $D$ ) or plaNETseq negative controls $(C)$. Pri-miRNAs were scaled from the beginning of miRNA-5p to the end of miRNA-3p ( $B$ and $C$ ) or using only the mature miRNAs sequences (D). Cyan and orange arrows indicated the processing fragments detected also marked in Figure 1. A scale identical to the plot displayed in Figure 2D is shown in the left panel of (C) while a zoom in is shown in the right panel.

(E) Co-transcriptional pri-miRNA processing score as measured by RTqPCR in RNAPII or IgG IP RNA-samples from Col-0, and hyl1-2. Error bars corresponds to 2xSEM. $\mathrm{P}<0.05\left({ }^{*}\right)$ or $<0.01\left(^{* *}\right)$, in a two-tailed unpaired T-Test, were considered statistically significant. RT-qPCR using primers pairs $A, B$ and $C$ in the IP fraction were normalized to U6 transcript and to the input levels using the same primers. Co-transcriptinal processing was measured as a reduction in the hairpin amplicon (primers $B$ ) relative to 5 ' region (amplicon A). Non-detected RNA levels are displayed as n.d. 

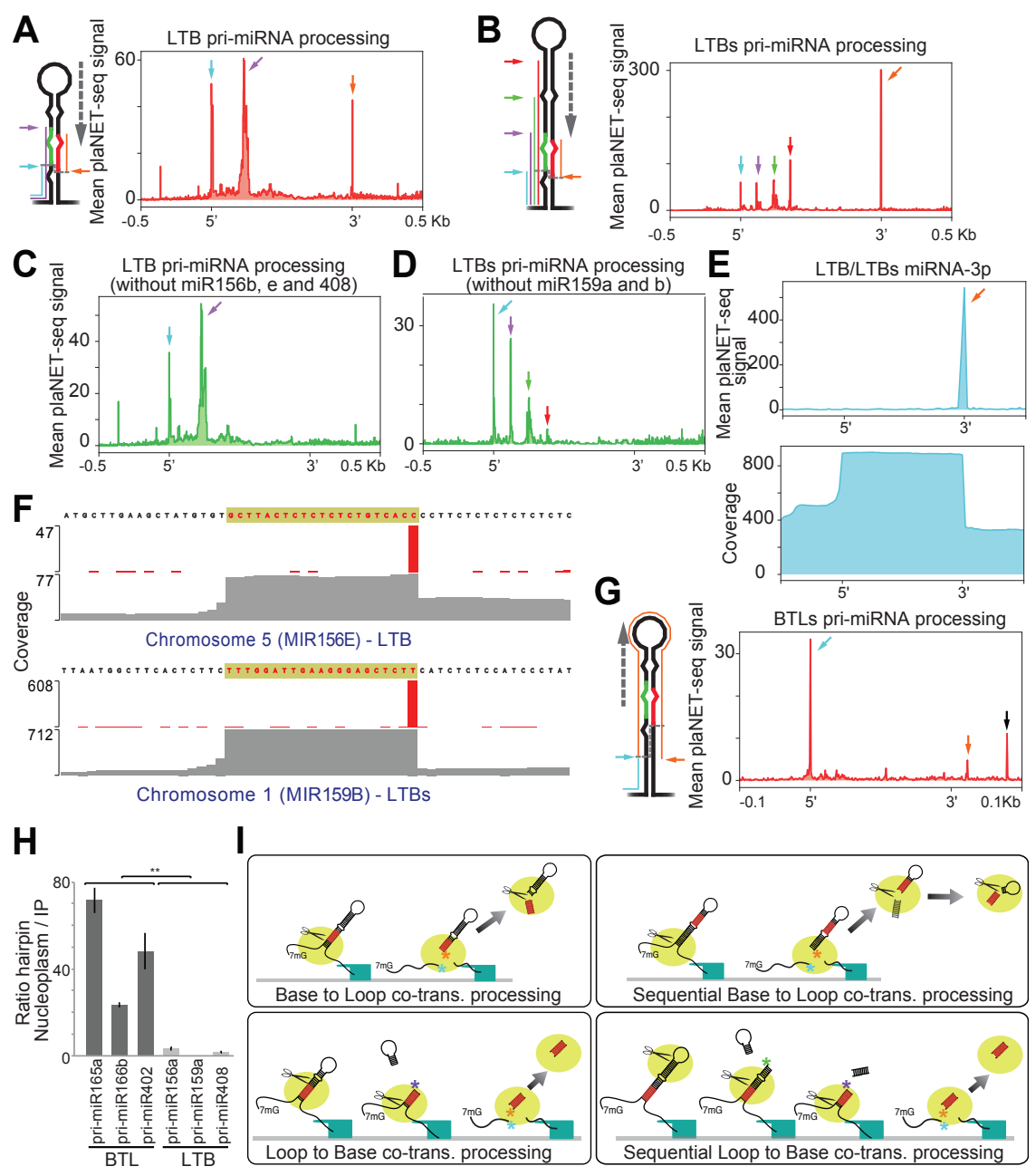

Figure 3. Co-transcriptional processing of BTL pri-miRNAs involves a second nucleoplasmic processing step.

$(A, B, C, D$, and $G$ ) Metagene analysis of processing intermediated on RNAPII-nascent LTB ( $A$ and $C$ ), LTBs ( $B$ and $D$ ), and BTLs $(G)$ pri-miRNAs as determined by scoring the 3'-end nucleotide of plaNET-seq reads. Pri-miRNAs were scaled from the beginning of miRNA-5p to the end of miRNA-3p. Colored arrows indicated the processing fragments detected for each processing type as illustrated in the schematic representation of LTB, LTBs, and BTLs pri-miRNAs. A peak corresponding to an exon donor site of $A T 1 G 12290$ is noted with a black arrow in panel $(\mathrm{G})$ and Figure S3F.

(E) Metagene analysis of plaNETseq LTB and LTBs scaled using the miRNA-3p sequence (top panel). Orange arrow matches the arrow in panel (B). Metagene of entire plaNETseq reads coverage over LTB and LTBs MIRNA loci, at the miRNA-3p encoding regions, showing the accumulation of 21 -nt long reads corresponding to the miRNA-3p mature sequences (bottom panel). 
(F) Mean plaNET-seq signal at the MIR156E and MIR159B loci (Red bars). Coverage of plaNET-seq reads over the same loci (Grey). The sequence corresponding to the mature miRNA-3p is noted on top of each panel highlighted in yellow.

(H) Relative abundance of BTL and LTB pri-miRNA hairpin region in the nucleoplasm compared to the RNAPII RIP fraction as measured by RT-qPCR in Col-0 samples. Error bars correspond to $2 x S E M . P<0.01{ }^{\left({ }^{*}\right)}$, in a two-tailed unpaired T-Test, were considered statistically significant. Processed hairpins measurements in the nucleoplasm and RNAPII IP fraction were normalized to the input levels for each samples. Quantifications were then expressed as a nucleoplasm/IP ratio of the values corrected by relative amount of unprocessed pri-miRNAs quantified with primers flanking the DCL1 cleavage site.

(I) Schematic summary of the co-transcriptional processing mechanisms of Arabidopsis pri-miRNAs. Color asterisks in each panel denote the plaNET-seq scored nucleotides for each processing type, and match the arrows of the same colors displayed in the previous panels and in Figure 2. 


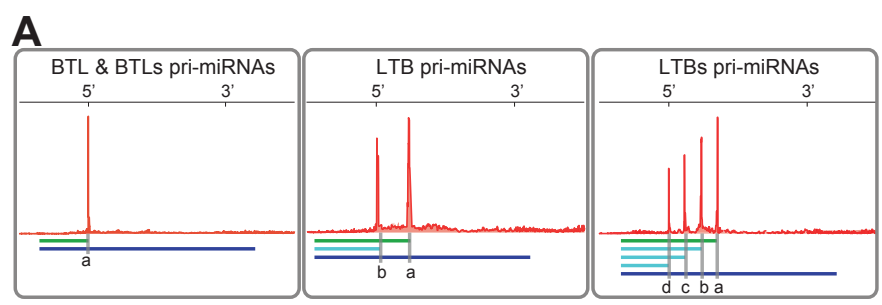

B
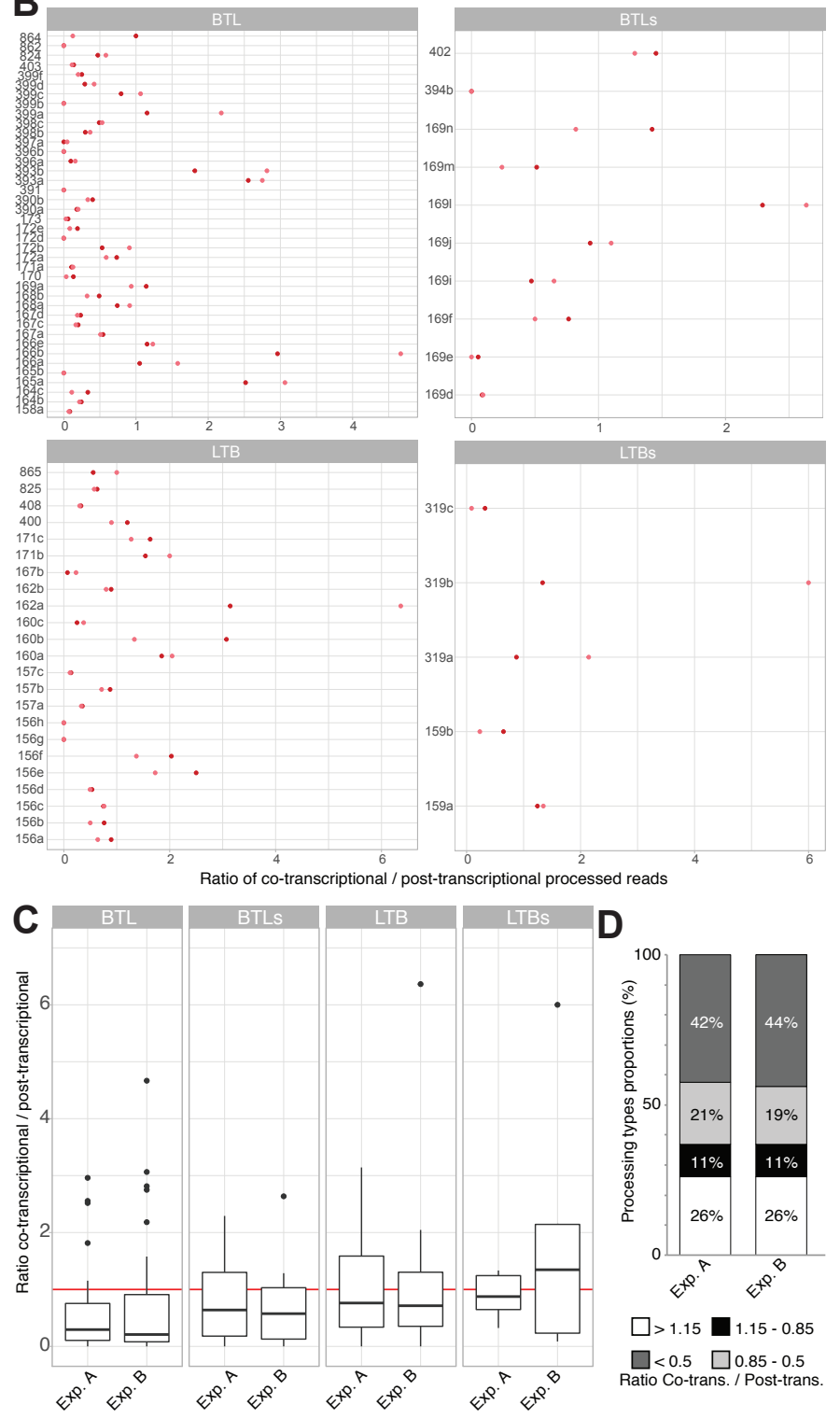

Figure 4. Co-transcriptionally and post-transcriptionally processing co-exist for most pri-miRNAs.

(A) Schematic representation of the positions used to quantify cotranscriptional vs post-transcriptional processing ratios. In all cases total number of reads ending in the nucleotide marked as (a) (green lines) were expressed relative to reads expanding this site (blue lines). 
(B) Co-transcriptional processing ratios corresponding to all analyzed miRNAs in two independent plaNET-seq experiments and split by processing mechanism.

(C) Box-plot representation of the co-transcriptional processing ratios. Red line mark ratio $=1$ where co- and post-transcriptinal processing are equally frequent.

(D) Fraction of pri-miRNAs preferentially processed co-transcriptionally, post-transcriptionally or with equal preference. Two independent experiments in control conditions and the addition of both are displayed. 

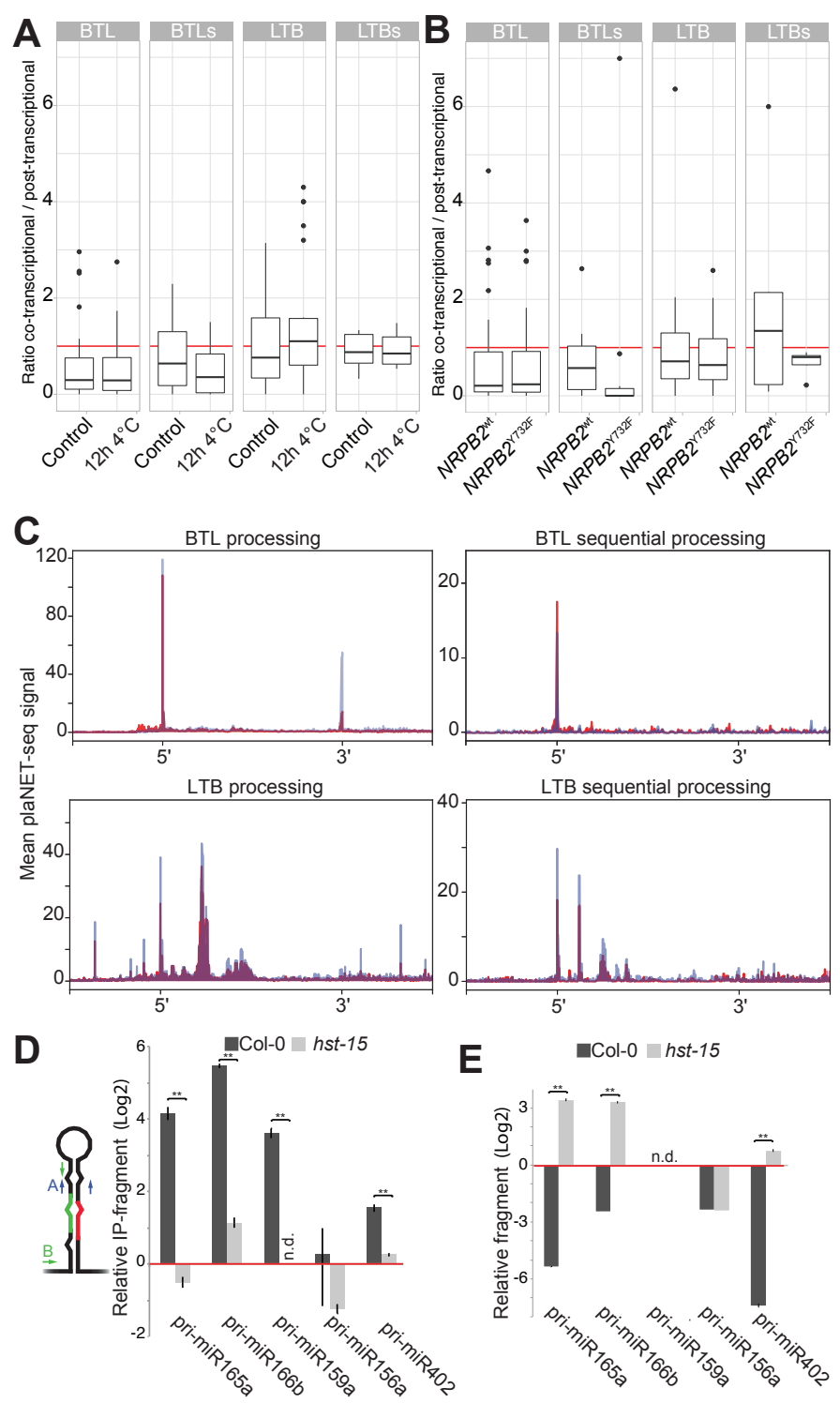

E
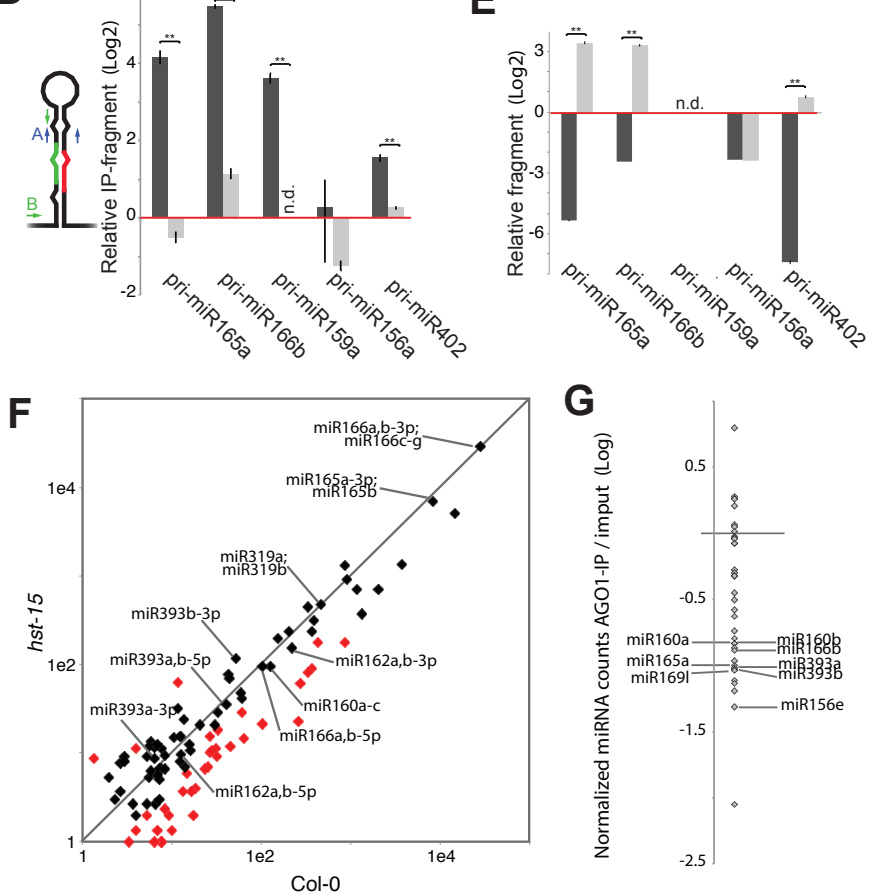

Figure 5. Co-transcriptional miRNA processing ratios are variable in different conditions

( $A$ and $B$ ) Box-plot representations of the co-transcriptional processing ratios in seedlings transfer to $4{ }^{\circ} \mathrm{C}$ for 12 hours or kept at control conditions (A) or nrbp2 mutant plants expressing a WT version of the proteins or a mutation 
(Y732F) that confers enhanced processivity to the RNAPII (C). Red line mark ratio $=1$ where co- and post-transcriptinal processing are equally frequent.

(E) Superposition of metagene analysis of plaNET-seq pri-miRNA processing intermediated in control (red) or NRPB2 ${ }^{\text {Y732F }}$ transgenic plants (Blue). Pri-miRNAs were scaled from the beginning of miRNA-5p to the end of miRNA$3 p$.

(F, and G) Pri-miRNA co-transcriptional processing in Col-0 and hst-15 mutant plants as measured by RT-qPCR in RIP $(F)$ or chromatin depleted nucleoplasm samples (G). Co-transcriptional processing was measured as the relative abundance of the hairpin region (Primers $A$ ) over the amount of unprocessed pri-miRNAs quantified with primers flanking the DCL1 cleavage site (Primers B). Processing intermediates were normalized by the input and expressed relative to the IgG IP samples (red line). Error bars corresponds to SEM. $\left.\mathrm{P}<0.01{ }^{* *}\right)$, in a two-tailed unpaired T-Test, were considered statistically significant.

(H) Scatter plot comparing the counts per million +1 (log scale) of mature miRNAs between wild type (Col-0) and hst-15 mutants. Differentially expressed miRNAs are shown in red. MiRNAs with the largest ratio of co-transcriptinal processing are noted individually.

(I) Dot-plot of AGO1-loaded miRNAs. MiRNAs in the AGO1-IP fraction as well as in the input samples were first expressed as a fraction of the total count of miRNAs in the respective sample. AGO1 loading preference for each miRNA is then expressed as the ratio of the frequency of a miRNA in the IP vs the input sample. MiRNAs with the largest ratio of co-transcriptinal processing are noted individually. 
bioRxiv preprint doi: https://doi.org/10.1101/2021.09.07.459282; this version posted September 7, 2021. The copyright holder for this

preprint (which was not certified by peer review) is the author/funder, who has granted bioRxiv a license to display the preprint in perpetuity. It is made available under aCC-BY-NC-ND 4.0 International license.

A

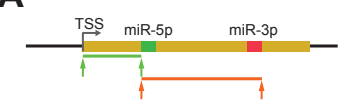

C

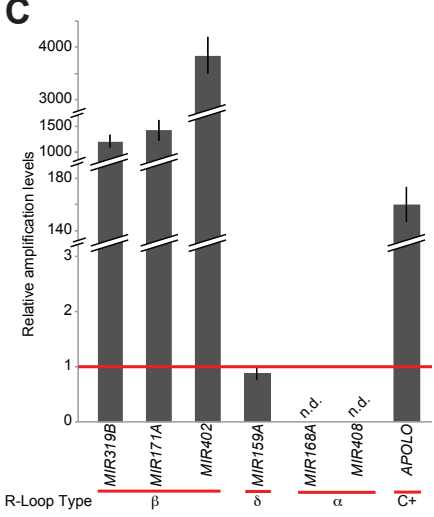

B

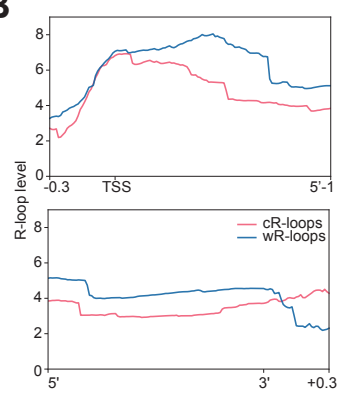

$E$

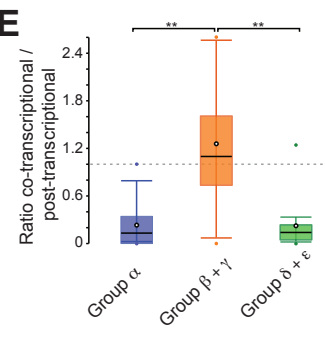

D
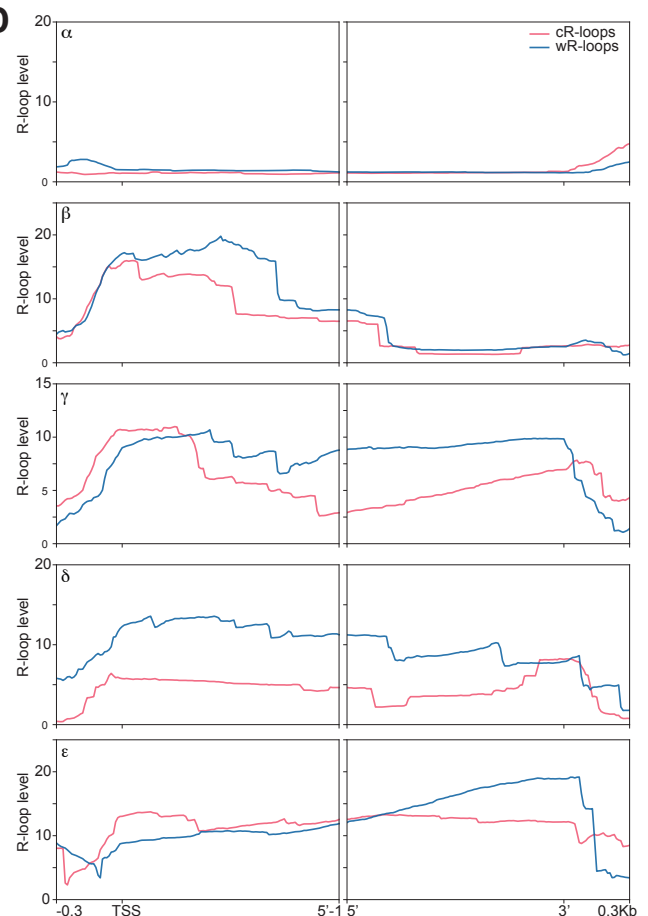
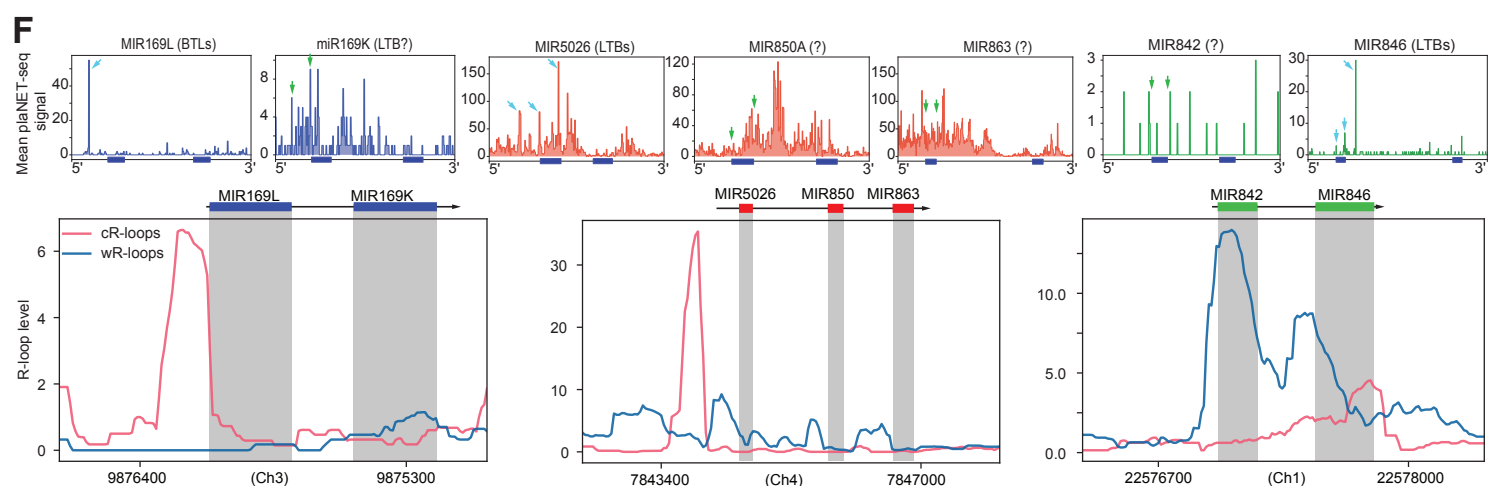

G
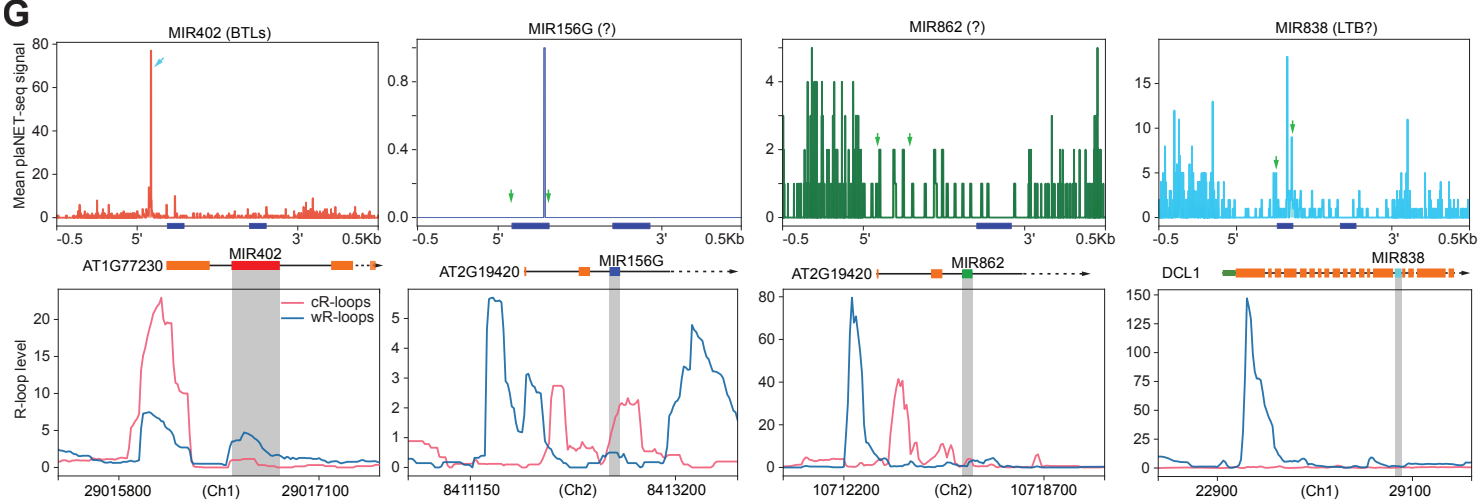

$\mathrm{H}_{0}$

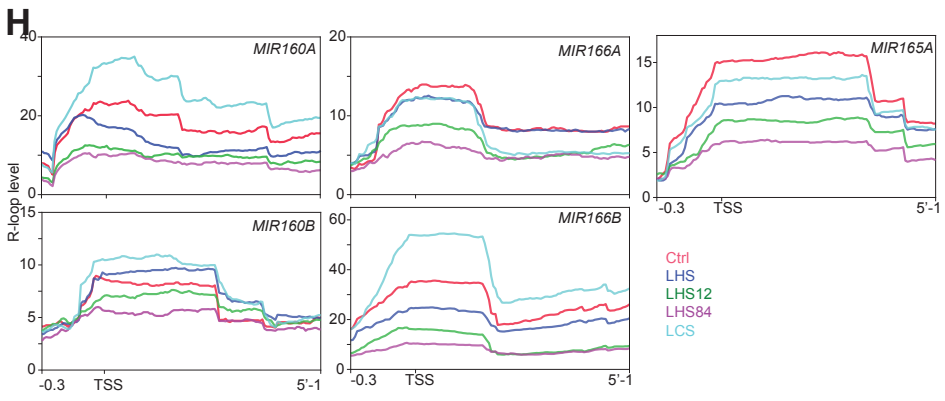

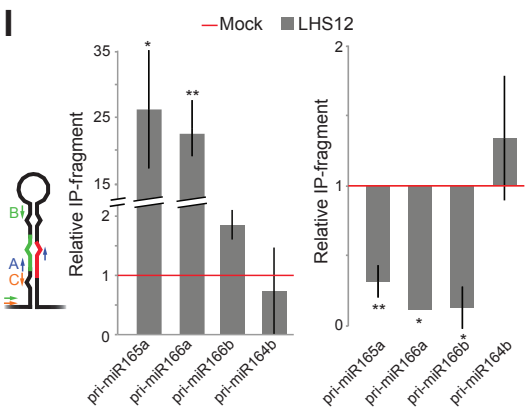


Figure 6. R-Loops at the 5' end of miRNA loci promotes co-

\section{transcriptional processing}

(A) Schematic representation of the region scaled for metagene analysis of R-Loop formation over MIRNA loci. MIRNA loci were scaled either from the transcription start site (TSS) to the nucleotide before miRNA-5p (green) or from miRNA-5p to the end of miRNA-3p (red). Metagene analysis was then plotted in ( $B$ and D) adding 300 bp upstream or downstream the windows respectively.

(B) Metagene analysis of R-loop formation over all MIRNA loci either in the Watson (blue) or Crick (red) strands.

(C) Quantification of R-loops near the TSS of MIRNAs belonging to different categories of R-loop pattern (defined in (D)) as measures by DRIP-qPCR assays. Values of DRIP samples are normalized to the input and expressed as relative to the DRIP signal in samples IP with a $\alpha-\lg$ antibody (red line). Failure to detect R-Loops are notes as non-detected (n.d.). Error bars correspond to 2xSEM.

(D) Metagene analysis of R-loop formation over MIRNA loci sorted by Rloop distribution. $\alpha$ - MIRNA loci without R-loops; $\beta$ - MIRNA loci with R-loops over the ssRNA 5 ' end of the pri-miRNAs; $\gamma$-MIRNA loci with bipartite R-loop signals at the beginning and end of the pri-miRNAs; $\delta$ - MIRNA loci with R-loops over the entire loci; $\varepsilon$ - MIRNA loci with colliding R-loops in the Watson (blue) and Crick (red) strands.

(E) Boxplot of co-transcriptional processing ratios of pri-miRNAs with $\mathrm{R}$ loop profiles of categories $\alpha, \beta+\gamma$, or $\delta+\varepsilon$. Equal processing type frequency (=1) is marked with a dashed line. Error bars shows the maximum and minimum values. $\left.P<0.01{ }^{* *}\right)$, in a two-tailed unpaired T-Test, were considered statistically significant.

$(F$, and $G)$ R-loop profile and plaNET-seq signals on polycistronic miRNA clusters $(F)$ and mirtron loci $(G)$. Cyan arrows in the plaNET-seq plots indicate accurate detected processing site. Green arrows mark the positions where peaks would be expected if the corresponding pri-miRNA are co-transcriptionally processed. The position of miRNA-5p and $-3 p$ are marked with blue boxed under the plaNET-seq plots. MiRNA precursor sequences within the containing locus are noted in gray within the R-loop profiles. 
(H) R-loop profile over individual miRNA loci as detected in samples extracted from control plants (Red line), plants incubated $30 \mathrm{~h}$ at $17^{\circ} \mathrm{C}$ (LCS) or at $37^{\circ} \mathrm{C}(\mathrm{LHS})$, plants incubated $30 \mathrm{~h}$ at $37^{\circ} \mathrm{C}$ and then returned to $23^{\circ} \mathrm{C}$ for 12 (LHS12) or 84 (LHS84) hours.

(I) Left panel: Co-transcriptional processing ratio in the H3 RIP fraction as measured as the accumulation of the hairpin region (Primers $A$ ) normalized to the unprocessed pri-miRNA (Primers B) by RT-qPCR. Right panel: Retention levels of processed 5'-end arms of pri-miRNAs (primers C) in the IP sample normalized by the input sample as measured by RT-qPCR. In both panels the quantification in the plants incubated for $30 \mathrm{~h}$ at $37^{\circ} \mathrm{C}+12 \mathrm{~h}$ at $12^{\circ} \mathrm{C}(\mathrm{LHS} 12$, grey bars) are expressed relative to the control samples (red line). Error bars corresponds to SEM. $P<0.01\left(^{* *}\right)$ and $\mathrm{P}<0.05\left(^{*}\right)$, in a two-tailed unpaired T-Test, were considered statistically significant. 